\title{
Lentiviral Hematopoietic Stem Cell Gene Therapy Benefits Metachromatic Leukodystrophy
}

Alessandra Biffi et al.

Science 341, (2013);

DOI: $10.1126 /$ science. 1233158

This copy is for your personal, non-commercial use only.

If you wish to distribute this article to others, you can order high-quality copies for your

colleagues, clients, or customers by clicking here.

Permission to republish or repurpose articles or portions of articles can be obtained by following the guidelines here.

The following resources related to this article are available online at www.sciencemag.org (this information is current as of November 7, 2013 ):

Updated information and services, including high-resolution figures, can be found in the online version of this article at:

http://www.sciencemag.org/content/341/6148/1233158.full.html

Supporting Online Material can be found at:

http://www.sciencemag.org/content/suppl/2013/07/10/science.1233158.DC1.html

A list of selected additional articles on the Science Web sites related to this article can be found at:

http://www.sciencemag.org/content/341/6148/1233158.full.html\#related

This article cites 58 articles, 15 of which can be accessed free:

http://www.sciencemag.org/content/341/6148/1233158.full.html\#ref-list-1

This article has been cited by 3 articles hosted by HighWire Press; see:

http://www.sciencemag.org/content/341/6148/1233158.full.html\#related-urls

This article appears in the following subject collections:

Medicine, Diseases

http://www.sciencemag.org/cgi/collection/medicine 


\section{Lentiviral Hematopoietic Stem Cell Gene Therapy Benefits Metachromatic Leukodystrophy}

Alessandra Biffi, ${ }^{*}$ Eugenio Montini, Laura Lorioli, Martina Cesani, Francesca Fumagalli, Tiziana Plati, Cristina Baldoli, Sabata Martino, Andrea Calabria, Sabrina Canale, Fabrizio Benedicenti, Giuliana Vallanti, Luca Biasco, Simone Leo, Nabil Kabbara, Gianluigi Zanetti, William B. Rizzo, Nalini A. L. Mehta, Maria Pia Cicalese, Miriam Casiraghi, Jaap J. Boelens, Ubaldo Del Carro, David J. Dow, Manfred Schmidt, Andrea Assanelli, Victor Neduva, Clelia Di Serio, Elia Stupka, Jason Gardner, Christof von Kalle, Claudio Bordignon, Fabio Ciceri, Attilio Rovelli, Maria Grazia Roncarolo, Alessandro Aiuti, Maria Sessa, Luigi Naldini*

Introduction: Metachromatic leukodystrophy (MLD) is a neurodegenerative lysosomal storage disease caused by arylsulfatase A (ARSA) deficiency. The disease primarily affects children and invariably leads to premature death. In previous work with a mouse model of MLD, we used a lentiviral vector (LV) to introduce a functional ARSA gene into hematopoietic stem cells (HSCs) ex vivo and showed that reinfusion of the engineered HSCs prevented and corrected disease manifestations in the animals. To determine whether this gene therapy strategy is safe and can offer therapeutic benefit to patients with early-onset MLD, we designed a phase I/II trial.

Methods: We optimized LV manufacturing and HSC transduction in clinical grade conditions. Three children with ARSA deficiency and mutations associated with early-onset MLD were treated at the presymptomatic stage; all had at least one older sibling affected by the same disease variant. HSCs from the patients were transduced ex vivo with a LV carrying the ARSA gene.

Patients were treated with a myeloablative busulfan conditioning regimen before reinfusion of the engineered HSCs. Clinical and objective evaluations were collected up to 24 months after treatment. Molecular follow-up of vector integration site distribution was performed on hematopoietic cells derived from bone marrow and peripheral blood.

Results: There was high-level stable engraftment of the transduced HSCs in the bone marrow and peripheral blood of all patients at all times tested, with 45 to $80 \%$ of bone marrow-derived hematopoietic colonies harboring the vector. With these high gene marking levels, ARSA activity was reconstituted to above normal values in the hematopoietic lineages and in the cerebrospinal fluid. Analysis of $>36,000$ different LV integration sites showed that the high gene marking was sustained by highly polyclonal engraftment of transduced cells without evidence of aberrant clonal behavior. Several integration sites were shared among progenitors and mature myeloid, B- and T-cells sampled at long intervals after treatment, indicating efficient transduction and engraftment of HSCs. These findings were associated with a clear therapeutic benefit because the disease did not progress in any of the treated patients, even after the time of onset projected from sibling cases.

Discussion: Our gene therapy protocol allows stable engraftment of transduced HSCs at high levels and without evidence of vector-induced genotoxicity. The reconstitution of ARSA activity in the cerebrospinal fluid and the arrested progression of neurodegenerative disease in the three treated patients demonstrate that the transplanted cells, or their progeny, can seed the nervous system and deliver therapeutic levels of active enzyme. Although our data are promising, long-term follow-up of the patients is needed in order to establish the full therapeutic potential of this gene therapy strategy for MLD. In addition, our data position LV gene transfer as a feasible means to engineer human hematopoiesis to its near entirety-an approach that could be exploited for treatment of other diseases.
READ THE FULL ARTICLE ONLINE

http://dx.doi.org/10.1126/science.1233158

Cite this article as $\mathrm{A}$. Biffi et al.

Science 341, 1233158 (2013).

DOI: $10.1126 /$ science. 1233158

\section{FIGURES IN THE FULL ARTICLE}

Fig. 1. Gene marking in patients after HSC-GT.

Fig. 2. ARSA expression in patients after HSC-GT.

Fig. 3. Clinical follow up of MLD patients after HSC-GT.

Fig. 4. LV genomic integration profile.

Fig. 5. Common insertion site analysis.

Fig. 6. Stem cell marking and clonal dynamics.

\section{SUPPLEMENTARY MATERIALS}

www.sciencemag.org/cgi/content/full/

science.1233158/DC1

Materials and Methods

Figs. S1 to S22

Tables S1 to S19

References

\section{RELATED ITEMS IN SCIENCE}

Aiuti et al., Lentiviral hematopoietic stem cell gene therapy in patients with Wiskott-Aldrich syndrome. Science 341, 1233151 (2013). doi:10.1126/science.1233151

I. Verma, Edging toward the promise of gene therapy. Science 341, 853-855 (2013).

doi:10.1126/science.1242551

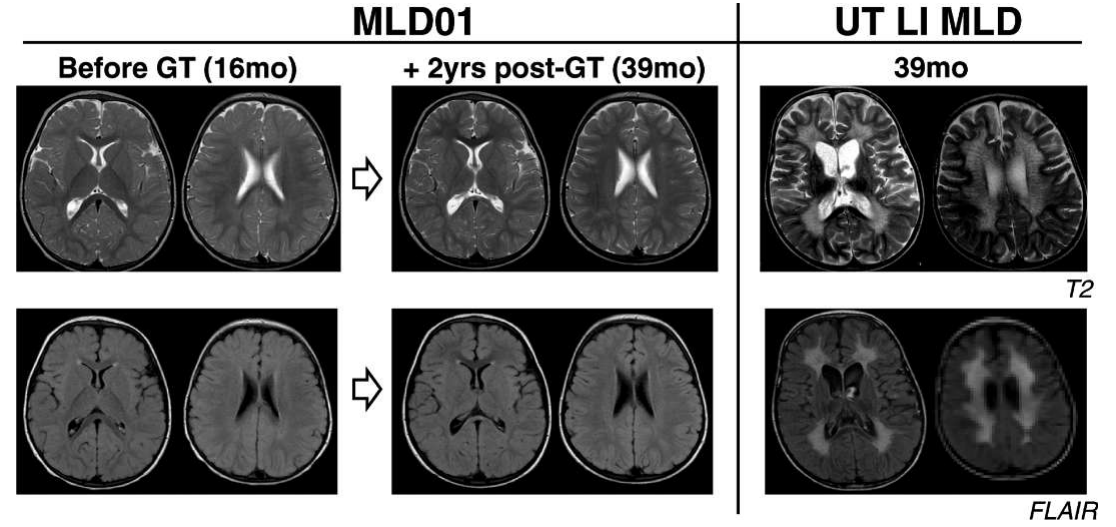

HSC gene therapy can prevent progression of metachromatic leukodystrophy. Magnetic resonance (MR) images of the brain of a patient (MLD01) before and after gene therapy. The brain of this patient appeared largely normal 2 years after treatment. In contrast, the brain of an untreated, agematched late infantile MLD patient (UT LI MLD) showed severe demyelination associated with diffuse atrophy. (Top) Axial T2 weighted fast spin-echo MR images. (Bottom) Fluid-attenuated inversion recovery (FLAIR) MR images. 


\section{Lentiviral Hematopoietic Stem Cell Gene Therapy Benefits Metachromatic Leukodystrophy}

Alessandra Biffi, ${ }^{1,2,3 *} \dagger$ Eugenio Montini, ${ }^{1 *}$ Laura Lorioli, $^{1,2,3,4}{ }^{\text {Martina Cesani, }}{ }^{1}$ Francesca Fumagalli, ${ }^{2,4,5}$ Tiziana Plati, $^{1}$ Cristina Baldoli, $^{6}$ Sabata Martino, ${ }^{7}$ Andrea Calabria, ${ }^{1}$ Sabrina Canale, ${ }^{2}$ Fabrizio Benedicenti, ${ }^{1}$ Giuliana Vallanti, ${ }^{8}$ Luca Biasco, ${ }^{1}$ Simone Leo, ${ }^{9}$ Nabil Kabbara, ${ }^{10}$ Gianluigi Zanetti, ${ }^{9}$ William B. Rizzo, ${ }^{11}$ Nalini A. L. Mehta, ${ }^{12}$ Maria Pia Cicalese, ${ }^{2,3}$ Miriam Casiraghi, ${ }^{2}$ Jaap J. Boelens, ${ }^{13}$ Ubaldo Del Carro, ${ }^{5}$ David J. Dow, $^{12}$ Manfred Schmidt, ${ }^{14}$ Andrea Assanelli, ${ }^{3,15}$ Victor Neduva, ${ }^{12}$ Clelia Di Serio, ${ }^{4}$ Elia Stupka, ${ }^{16}$ Jason Gardner, ${ }^{17}$ Christof von Kalle, ${ }^{14}$ Claudio Bordignon, ${ }^{4,8}$ Fabio Ciceri, ${ }^{3,15}$ Attilio Rovelli, ${ }^{18}$ Maria Grazia Roncarolo, ${ }^{1,2,3,4}$ Alessandro Aiuti, ${ }^{1,2,3,19}$ Maria Sessa, ${ }^{2,5}$ Luigi Naldini $^{1,4} \dagger$

Metachromatic leukodystrophy (MLD) is an inherited lysosomal storage disease caused by arylsulfatase A (ARSA) deficiency. Patients with MLD exhibit progressive motor and cognitive impairment and die within a few years of symptom onset. We used a lentiviral vector to transfer a functional ARSA gene into hematopoietic stem cells (HSCs) from three presymptomatic patients who showed genetic, biochemical, and neurophysiological evidence of late infantile MLD. After reinfusion of the gene-corrected HSCs, the patients showed extensive and stable ARSA gene replacement, which led to high enzyme expression throughout hematopoietic lineages and in cerebrospinal fluid. Analyses of vector integrations revealed no evidence of aberrant clonal behavior. The disease did not manifest or progress in the three patients 7 to 21 months beyond the predicted age of symptom onset. These findings indicate that extensive genetic engineering of human hematopoiesis can be achieved with lentiviral vectors and that this approach may offer therapeutic benefit for MLD patients.

$\mathrm{M}$ etachromatic leukodystrophy (MLD) is an autosomal recessive lysosomal storage disease caused by mutations in the ARSA gene that result in a deficiency of the enzyme arylsulfatase A (1). ARSA deficiency causes accumulation of the enzyme substrate sulfatide in oligodendrocytes, microglia, and certain neurons of the central nervous system (CNS), and in Schwann cells and macrophages of the peripheral nervous system (PNS). This build-up of sulfatide leads to widespread demyelination and neurodegeneration, which is ultimately manifested in patients as severe progressive motor and cognitive impairment. MLD is classified into clinical variants according to the age of symptom onset. Patients with the late-infantile (LI) variant show symptoms within the second year of life, have the most severe manifestations of the disease, and die within a few years of symptom onset. Current treatments do not substantially delay the progression or fatal outcome of $\operatorname{MLD}(2,3)$.

Hematopoietic stem cell transplantation (HSCT) and HSC gene therapy (HSC-GT) have been investigated for the treatment of lysosomal storage diseases because enzyme-proficient hematopoietic progeny cells can migrate to the affected tissues, including the CNS, scavenge the stored material, such as sulfatide in MLD, and cross-correct the resident cells (4). The efficacy of these treatments varies among different types of lysosomal storage diseases $(3,5-7)$ and with the extent of functional gene expression in the transplanted cells $(8-11)$.
Achieving a high level of functional gene replacement is particularly important in the setting of HSC-GT; to date, gene replacement in the hematopoietic cells of treated patients has been limited (12-15).

In a mouse model of MLD, we have demonstrated that disease manifestations can be prevented and corrected with lentiviral vector (LV)-based HSC-GT but not HSCT $(8,9,16)$. This is consistent with the observation that HSCT fails to provide consistent benefits in MLD patients (3, 5-7). LV-based HSC-GT induced extensive and supraphysiological expression of the functional ARSA gene throughout the HSC progeny, which in turn mediated widespread crosscorrection of CNS and PNS resident cells $(8,9)$.

Translating this strategy into a clinical protocol, however, posed several challenges, including gene transfer efficacy and safety. Although stable marking ( 10 to $15 \%$ ) was reported in two patients with X-linked adrenoleukodystrophy (X-ALD) in the first trial of LV-mediated HSCGT (17), it was not clear whether LVs could transduce human HSCs in clinically relevant settings with the efficiency required for application to MLD gene therapy. Vector safety is also a concern in light of the clinical experience with $\gamma$-retroviral vectors, which showed that genome-wide vector integration in hematopoietic stem/progenitor cells (HSPCs) has the potential to trigger leukemia through insertional mutagenesis $(14,18)$. Although several studies have shown that LVs reduce the risk of genotoxic insertions because of their advanced design and the integration site selection typical of the parental HIV $(19,20)$, it remained possible that increasing the total integration load in the infused HSPCs would offset the biosafety advantage.

In the present study, we optimized LV-mediated gene transfer into human HSCs for clinical translation and used this HSC-GT protocol to treat nine patients with early onset MLD in a phase I/II trial. Here, we report the outcome of the treatment in the first three treated patients at 24 months follow-up for patient MLD01 and 18 months follow-up for patients MLD02 and MLD03.

Results

\section{Efficient ex Vivo Transfer of the ARSA Gene} into the HSPCS of MLD Patients

Transduction of human bone marrow (BM)derived $\mathrm{CD} 34^{+}$cells was optimized to reach $\geq 2$ vector copy number per genome (VCN) (fig. S1), based on the ARSA overexpression levels required for therapeutic efficacy in preclinical studies $(8,9,21,22) . \mathrm{CD} 34^{+}$cells were stimulated ex vivo with early acting cytokines in serum-free medium and transduced with purified third-generation LVs encoding the human ARSA cDNA under the control of the human phosphoglycerate kinase promoter (PGK) (fig. S2). VCN was measured in the progeny of the treated cells obtained by means of bulk liquid culture, colony-forming assays and repopulation of chimeric $\mathrm{Rag}^{-/-} \mathrm{\gamma chain}^{-/-}$mice.

${ }^{1}$ San Raffaele Telethon Institute for Gene Therapy (TIGET), San Raffaele Scientific Institute, 20132 Milan, Italy. ${ }^{2}$ TIGET Pediatric Clinical Research Unit, Division of Regenerative Medicine, Stem Cells and Gene Therapy, San Raffaele Scientific Institute, 20132 Milan, Italy. ${ }^{3}$ Pediatric Immunohematology and Bone Marrow Transplant Unit, San Raffaele Scientific Institute, 20132 Milan, Italy. ${ }^{4}$ Vita-Salute San Raffaele University, 20132 Milan, Italy. ${ }^{5}$ Neurology Unit, Department of Neurology, San Raffaele Scientific Institute, 20132 Milan, Italy. ${ }^{6}$ Neuroradiology Unit, Head and Neck Department, San Raffaele Scientific Institute, 20132 Milan, Italy. ${ }^{7}$ Department of Experimental Medicine and Biochemical Sciences, University of Perugia, 06122 Perugia, Italy. ${ }^{8}$ MolMed, 20132 Milan, Italy. 'Distributed Computing Group, Center for Advanced Studies, Research and Development in Sardinia (CRS4), 09010 Pula, Italy. ${ }^{10}$ Pediatric Hematology Oncology Division, Rafic Hariri University Hospital, Beirut, Lebanon. ${ }^{11}$ Department of Pediatrics, University of Nebraska Medical Center, Omaha, NE 68198, USA. ${ }^{12}$ Molecular and Cellular Technologies, GlaxoSmithKline, Stevenage 5G1 2NY, UK. ${ }^{13}$ Pediatric Blood and Marrow Transplantation Program, University Medical Center 3584 CX Utrecht, Netherlands. ${ }^{14}$ Department of Translational Oncology, National Center for Tumor Diseases and German Cancer Research Center, 69120 Heidelberg, Germany. ${ }^{15} \mathrm{He}-$ matology and Bone Marrow Transplant Unit, San Raffaele Scientific Institute, 20132 Milan, Italy. ${ }^{16}$ Center for Translational Genomics and Biolnformatics, San Raffaele Scientific Institute, 20132 Milan, Italy. ${ }^{17}$ Regenerative Medicine Discovery Performance Unit, GlaxoSmithKline Research and Development, King of Prussia, PA 19406, USA. ${ }^{18}$ Bone Marrow Transplant Unit, MBBM Foundation, Pediatric Department, Milano-Bicocca University at San Gerardo Hospital, 20052 Monza, Italy. ${ }^{19}$ University of Rome Tor Vergata, 00133 Rome, Italy.

*These authors contributed equally to this work. †Corresponding author. E-mail: biffi.alessandra@hsr.it (A.B.); naldini.luigi@hsr.it (L.N.) 
A summary of the LV manufacturing process (flow chart, main steps, and yields) as well as the specifications set for testing the good manufacturing practice (GMP) lots released for this study are reported in fig. S3 and tables S1 and S2.

After optimizing the gene transfer procedures for human cells, we designed a clinical trial of HSC-GT in which autologous HSPCs from patients with MLD would be transduced ex vivo with ARSA-encoding LVs and then reinfused after the patients had been treated by a myeloablative regimen using the alkylating agent busulfan (fig. S4). Because MLD patients usually express residual, nonfunctional ARSA protein, they are unlikely to mount an immune response toward ARSA. Our trial design therefore did not include immunosuppressive agents.

Three presymptomatic MLD patients, biochemically characterized for ARSA deficiency and carrying mutations associated with LI MLD, were enrolled and treated; all patients had one or more older siblings with LI MLD onset within 2 years of age (table S3). At enrollment, patients were defined as presymptomatic based on the lack of obvious clinical signs of the disease, independent of the presence of abnormalities in instrumental tests [electroneurographic recordings (ENG) and/or brain magnetic resonance imaging (MRI)]. Treatment of the patients began 2 to 12 months before the reported age of onset of the disease in their affected matched siblings (23-25).

Twenty to 30 days after collection of an HSC back-up, a BM harvest was performed followed by isolation of $\mathrm{CD} 34^{+}$cells by means of standard immunomagnetic procedures. Transduction of patients' $\mathrm{CD} 34^{+}$cells was performed as described above. Cells were washed, resuspended, and kept at $+4^{\circ} \mathrm{C}$ until successful completion of a first panel of fast quality control (QC) tests (table S4). The transduced $\mathrm{CD} 34^{+}$cells were then released and infused fresh intravenously. Other relevant QC results (table S4) became available 2 to 5 weeks after infusion. VCN ranged from 2.5 to 4.4 , transduction efficiency was 90 to $97 \%$, and ARSA activity was reconstituted at $\geq 10$-fold the level measured in healthy controls in the cultured progeny of the infused cells (fig. S1 and table S5).

A myeloablative, dose-adjusted busulfan regimen [target area under the curve (AUC) 4800 $\mu \mathrm{g} / \mathrm{L} *$ hour] (table $\mathrm{S} 5$ ) was administered intravenously to the patients from day -4 up to day -1 before HSC-GT in a total of 14 doses, with the last busulfan dose administered $\geq 24$ hours before transduced cell infusion. The conditioning regimen was well tolerated. The patients experienced severe neutropenia (absolute neutrophil count $<500 / \mu \mathrm{l}$ ) from as early as day +9 up to day +45 after transplant at the latest (fig. S5 and table S5). No reduction or only a minor transient reduction of lymphocyte counts was observed in the absence of immunosuppressive/lymphotoxic drugs in the pretransplant conditioning (fig. S5). Patients required transfusional support up to day +45 after treatment for thrombocytopenia and anemia (fig. S5). Thereafter, the patients rapidly recovered he- matological parameters matching the normal values for age. No abnormal expansion or clonal outgrowth was detected in the peripheral blood (PB) and BM by cell type composition, cytologic, karyotypic, or immunophenotypic studies (fig. S5). A mild (maximum National Cancer Institute/ National Institutes of Health common toxicity criteria grade 2) transient increase in hepatocellular enzyme levels was detected within 2 to 3 weeks after treatment. Serious adverse events included two cases of central venous catheter-related infection, which promptly resolved upon antibiotic treatment. Replication competent LVs (RCLs), antibodies to HIV Gag p24, and antibodies to ARSA in patients' $\mathrm{PB}$ were negative at all times tested.

\section{High-Level Engraftment of the Transduced HSPCS and Sustained ARSA Reconstitution}

Beginning 1 month after transplant, we observed high-level stable engraftment of the transduced cells in the BM and $\mathrm{PB}$ of all patients at all times tested (Fig. 1A). Between 45 and $80 \%$ of the colonies outgrown from patients' BM in the colonyforming cell (CFC) assay harbored the LV genome (Fig. 1A). Quantitative polymerase chain reaction (PCR) on $\mathrm{CD} 34^{+}$cells sorted from the patients' BM showed stable gene marking with $\mathrm{VCN}$ ranging from 0.9 to 1.9 up to the latest followup time (Fig. 1B). Considering the fraction of cells harboring the vector (estimated from the percentage of clonogenic progenitors outgrown from the patients' BM positive for LV DNA), these values imply a VCN of 2 to 4 in the engrafted transduced $\mathrm{CD} 34^{+}$cells, which closely
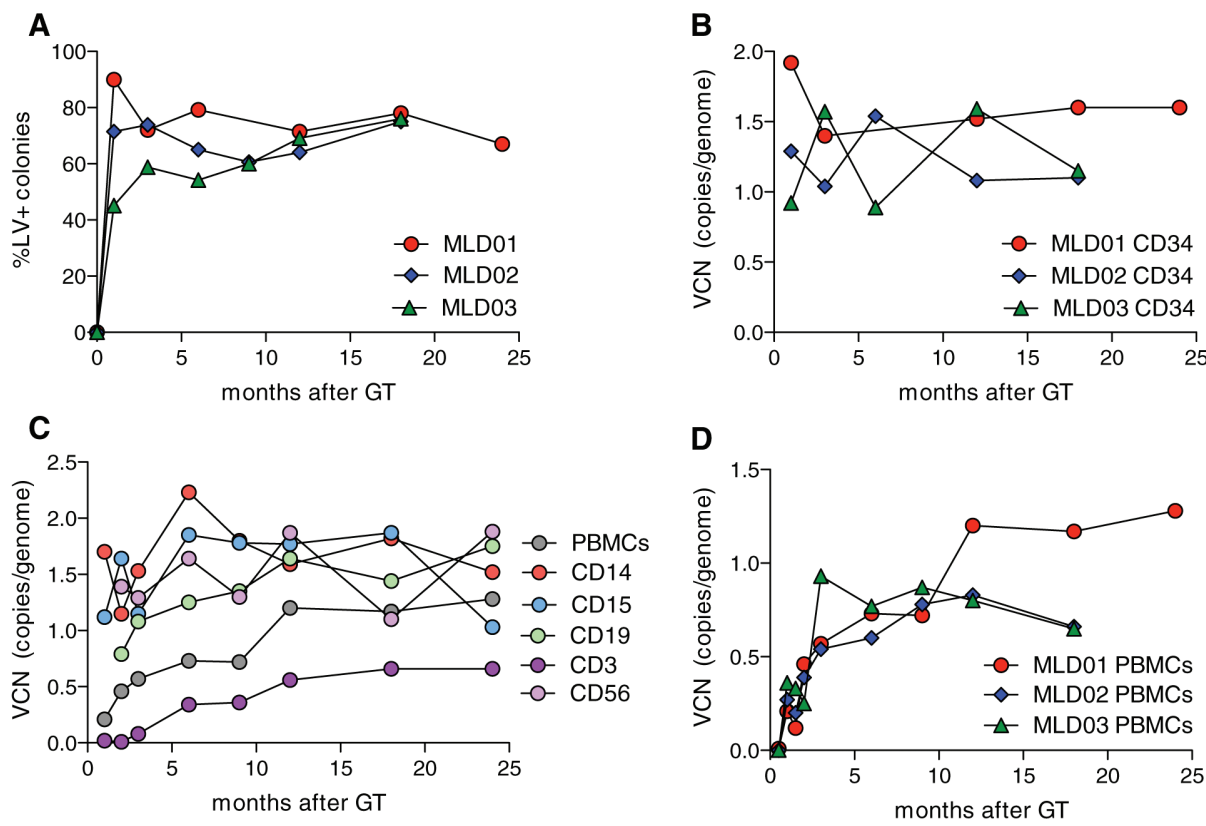

Fig. 1. Gene marking in patients after HSC-GT. (A) Engraftment of the transduced cells, evaluated with quantitative PCR on individual colonies from CFC assay performed on PB- and BM-derived cells and expressed as percentage (\%) of $\mathrm{LV}^{+}$colonies on total tested colonies. (B to $\left.\mathbf{D}\right)$ VCN expressed as copies of LV/human genome measured with quantitative PCR on BM-derived $\mathrm{CD}_{3} 4^{+}$cells (B), individual subpopulations isolated from PB of patient MLD01 (C), and total PBMCs from patients MLD01, -02 and -03 (D). 
tional ARSA was isolated from cerebrospinal fluid (CSF) collected from all three patients 1 year (and 2 years for MLD01) after HSC-GT at levels and activity comparable with those obtained from healthy donor samples, whereas no ARSA protein could be isolated from CSF before HSC-GT treatment (Fig. 2, C and D). These data demonstrate stable above-normal ARSA expression throughout the hematopoietic lineages and efficient delivery and bioavailability of the enzyme in the CNS after HSC-GT.

\section{HSC-GT Provides Therapeutic Benefit to MLD Patients}

Clinical observation and objective evaluations were collected up to 24 months after treatment for MLD01 and up to 18 months after treatment for MLD02 and MLD03. At the last follow-up, MLD01, MLD02, and MLD03 were 39, 30, and 25 months old, respectively (table S3). Because disease onset in the LI variant occurs invariably before 24 months of age and clinical manifestations are highly homogeneous, in particular among siblings $(23,24)$, all three patients in our study were evaluated beyond their expected age of disease onset.

MLD01 is particularly informative because the follow-up time for this patient is longest and because the predicted time of symptom onset was earlier than that of the other two patients. This patient's two older siblings manifested regression of psychomotor performance and arrest of acquisition of new motor and cognitive skills at 18 months of age. Consistent with LI-MLD natural history (26), both siblings had rapid disease progression after onset and reached level 6 of the Gross Motor Function Scale for MLD (GMF-C MLD) (27) by 30 months of age, when they were wheelchairbound and unable to support their head and trunk (table S7). In contrast, at 39 months of age MLD01 was able to stand independently and to walk and run with single aid (either the hand of another individual or an external walking device) (level 2 of the GMF-C MLD scale) (table S7). His Gross Motor Function Measure (GMFM) score progressively increased from enrollment (GMFM score of 164) up to a score of 193 at 39 months, which was substantially higher than all the scores recorded in our age-matched historical LI-MLD cohort [5.5 on average (26)], suggesting a continuous motor development (Fig. 3A and table S8). Cognitive evaluation by the Bayley Scale of Infant and Toddler Development revealed a normal composite IQ score for chronological age, with normal language and cognitive abilities (table S9) at all tested time points, including the last one at 39 months of age, an age at which the two affected siblings were incapable of any voluntary speech. The preexisting severe peripheral neuropathy of MLD01 [nerve conduction velocity (NCV) index $(26)-11.5$ at baseline] improved after treatment (NCV index -7.1 at last follow-up) (Fig. 3B). Brain MRI showed slight signal inhomogeneity, present since baseline evaluation and stable thereafter, and a small area of hyper-intensity in the corpus callosum that appeared at the 12-month follow-up and remained stable thereafter. All other brain MR findings were normal for age (Fig. 3C). In contrast, untreated LI-MLD patients invariably develop extensive and severe demyelination associated with diffuse atrophy by 24 to 36 months of age (Fig. 3C) $(26,28)$. To our knowledge, no unequivocally diagnosed LI-MLD patient at the age of 39 months has been reported to display clinical features as positive as those we observed in MLD01.

Patients MLD02 and MLD03 remain fully asymptomatic, with normal motor and cognitive development for their ages (30 and 25 months, respectively); however, the clinical follow-up period for these patients after the predicted time of symptom onset was shorter than that for MLD01. At the latest clinical follow-up (18 months after treatment), MLD02 was 7 months beyond and MLD03 was 10 months beyond the predicted time of symptom onset. Both patients had a GMF-C MLD score of 0 at the last evaluation, which is in sharp contrast with the disease evolution documented in their affected siblings at the corresponding age (table S7). Their GMFM scores progressively increased after treatment, which is consistent with the acquisition of normal developmental motor milestones for age (Fig. 3A and table S8). Furthermore, in both patients the Bayley Scale of Infant and Toddler Development showed a normal composite IQ score for the age at all times tested (table S9). The ENG findings for both patients remained stable from baseline up to the latest follow-up; MLD02 showed near normal motor and sensory conduction velocities since baseline, whereas MLD03 showed a stable mild demyelinating neuropathy (Fig. 3B). Brain MRI revealed that both patients had a normal progression of white matter signal due to myelination up to the latest follow-up. MLD02 had small areas of T2 and fluid-attenuated inversion recovery (FLAIR) hyper-intensity signal in posterior and anterior periventricular white matter, present since baseline and stable up to the latest follow-up (fig. S9).
Fig. 2. ARSA expression in patients after HSC-GT. (A and B) ARSA activity measured with the $p$-nitrocatechol sulfate (PNC) assay on CD15 ${ }^{+}$ cells (A) and CD14 ${ }^{+}$cells (B) isolated from the patients' $\mathrm{PB}$. The activity range measured in a cohort of healthy donors (HDs) ( $n \geq 10$ subjects) is shown. (C) Representative DEAEcellulose-chromatography analysis on $500 \mu$ l of cerebrospinal fluid (CSF) from a pool of four HDs, of a representative MLD patient before treatment (MLD01 pre-GT) and of the same patient 1 year after gene therapy. The peak of activity corresponds to the native form of the ARSA enzyme, as also demonstrated by its absence/very low residual activity in the patient's pretreatment sample. (D) Specific activity (toward MUS) of the ARSA enzyme isolated from the CSF of HDs (two cohorts of four donors each) and of the treated MLD patients (circles, MLD01; blue diamond, MLD 02; and green triangle, MLD03) sampled 12 months (red circle, MLD01; MLD02 and -03 were also sampled at 12 months) and 24 months (pink circle, MLD01) after treatment.
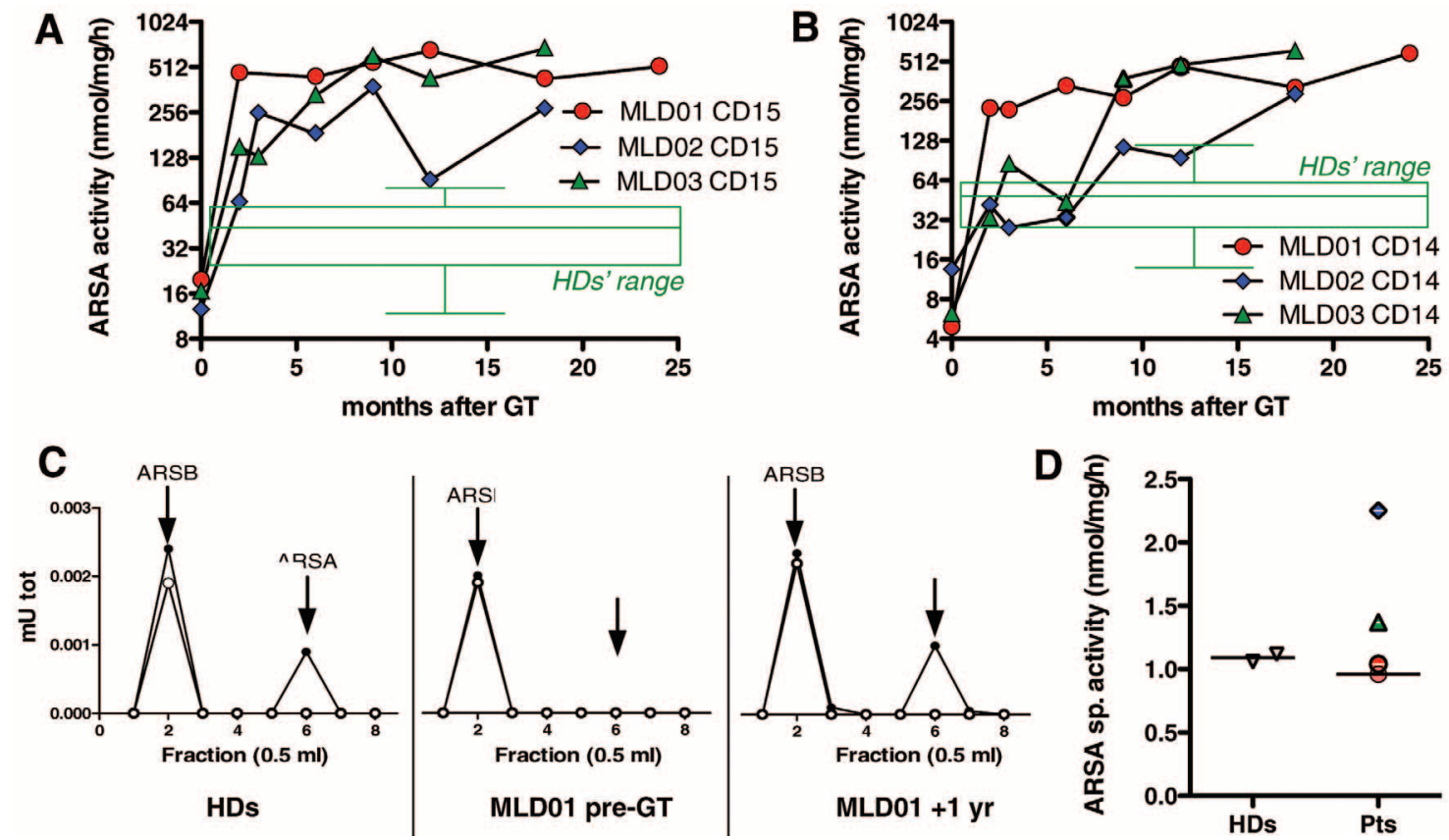


\section{Polyclonal Reconstitution of Hematopoiesis Without Evidence of Vector Genotoxicity}

To determine whether there was any cause for concern regarding the safety of the LV in these patients, we monitored the clonal dynamics of hematopoietic repopulation at the molecular level by analyzing the vector genomic integrations in the patients' reconstituted hematopoietic cells (figs. S10 and S11, table S10, and materials and methods). Because of the genome-wide quasi-random integration profile of $\mathrm{LV}$, each insertion creates a different genetic marker that can be used to track the clonal behavior of individual transduced cells. Genomic DNA was obtained from whole BM,
PB cells, and subsets (table S11) at 1, 3, 6, 9, and 12 months for all patients and, for MLD01 and MLD02, 18 months after HSC-GT (table S12). Vector-genome junctions were retrieved by means of linear amplification mediated-PCR (LAM-PCR) by using three different restriction enzymes (fig. S12). From the three patients, $>800$ independent LAM-PCRs were generated and sequenced by 454-Roche (Basel, Switzerland) and Illumina (San Diego, California) technologies to obtain $>10 \times 10^{6}$ sequence reads. Using a bioinformatics analysis pipeline and collision/contamination filtering (fig. S10, fig. S13, and table S13, A to C), we mapped integration sites (IS) for patient MLD01 $(1,120,414)$,

A

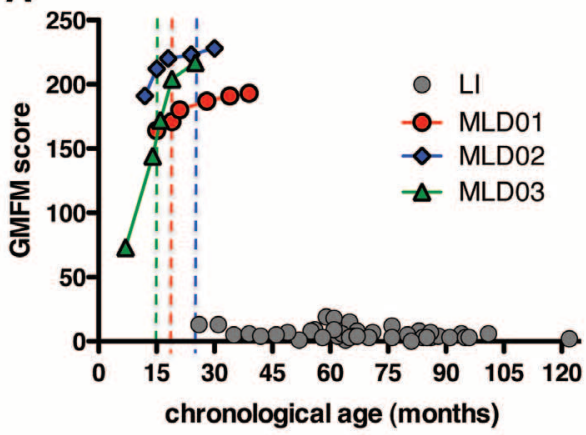

B

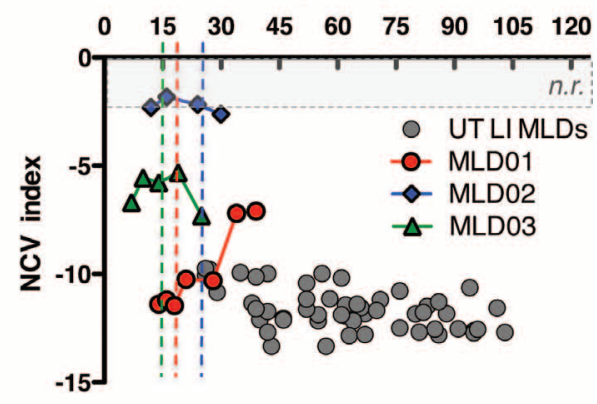

C

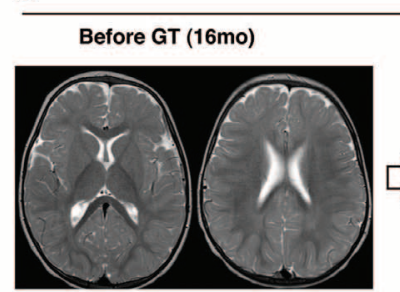

MLD01
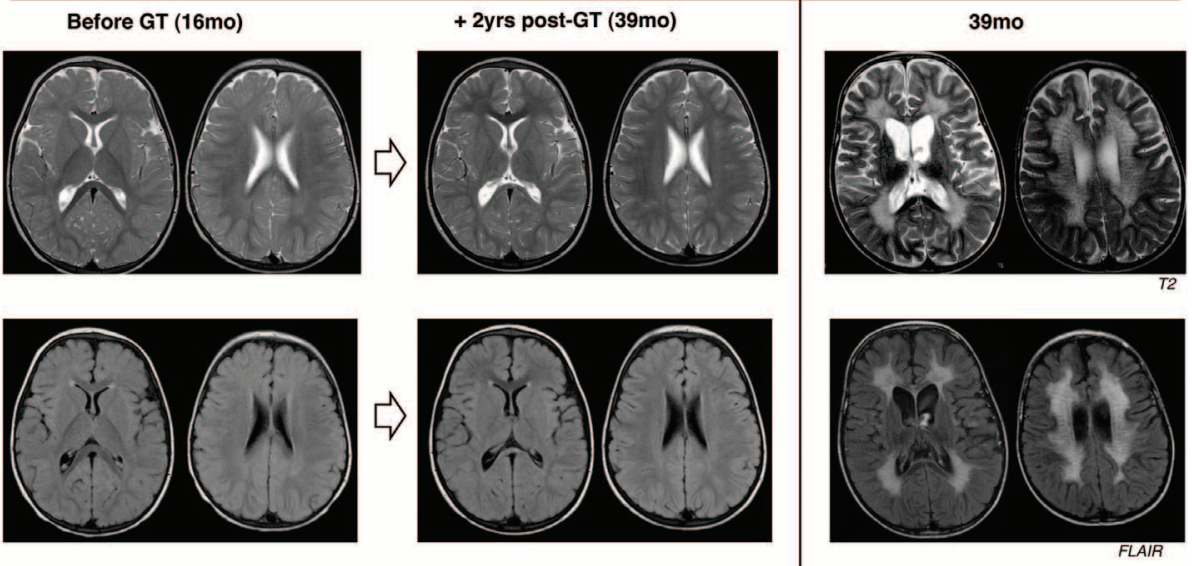

Fig. 3. Clinical follow up of MLD patients after HSC-GT. (A and B) GMFM score (A) and NCV index (B) of the three treated patients and of a historical cohort of LI-MLD patients (gray circles). The dotted lines indicate for each treated patient (inset, color code) the expected time of disease onset, according to the disease onset observed in their affected matched siblings; n.r., normal range of the NCV index. (C) Axial T2 weighted fast spin-echo MR images (top) and FLAIR MR images (bottom) obtained from patient MLD01 at baseline (before GT) and at +2 years after treatment, and corresponding (equivalent) images of an agematched untreated patient with LI-MLD (in parenthesis, the chronological age at imaging acquisition in months). In MLD01 images, a small area of hyperintensity is present within the splenium of the corpus callosum, stable in extension and appearance as compared with that in the +12 months follow-up; subtle signal inhomogeneities are present within posterior periventricular and posterior centrum semiovale white matter, in the absence of focal lesions; these inhomogeneities, present since baseline as just barely T2 and FLAIR hyperintensity signal, are (now) more evident because of the normal signal of the surrounding myelin; subarachnoid and ventricular spaces are within normal limits, even if a little wider when compared with that of the baseline. Basal ganglia and thalami remain of normal appearance. In UT LI-MLD images, extensive, diffuse symmetric hyperintensities with typical "tigroid pattern" are seen within periventricular white matter, centrum semiovale, corpus callosum, external and internal capsules, and cerebellar deep white matter. A severe diffuse brain atrophy involving basal ganglia and thalamy, which show $\mathrm{T} 2$ hypointense signal, is also present.
MLD02 (1,518,802), and MLD03 $(425,356)$ on the human genome (NCBI HG19). Because many identical ISs were retrieved from multiple lineages and/or time points, the number of distinct ISs was $14,482,11,077$, and 10,959 for patients MLD01, MLD02 and MLD03, respectively. The genomic distribution of IS, both in the in vitrocultured $\mathrm{CD} 34^{+}$cells and in vivo, matched the previously reported LV preference for integration within transcriptional units (on average, $80 \%$ of IS were within genes) (fig. S14). To identify the gene classes preferentially targeted by LV integrations, we analyzed IS data sets from either the in vitro progeny of the infused $\mathrm{CD} 34^{+}$cells or a pool of all in vivo-harvested samples for each patient using the GREAT (Genomic Regions Enrichment of Annotations Tool) software (table S14, A to F). In all patients, the gene classes of chromatin modification/remodeling, major histocompatibility complex class II-related functions, steroid hormone receptors, and RNA processing were overrepresented (table S14, A to F). These gene classes were the same, or shared $>75 \%$ genes, with gene classes overrepresented in the ALD HSC-GT $(17,29)$ (Fig. 4A and fig. S15).

We measured the proportion of sequencing reads representing each IS within our data sets as a surrogate readout for the relative abundance of the cell clone harboring that integration at a given time. For each time point, we analyzed the IS data sets from the $\mathrm{CD} 34^{+}$, myeloid (pooled $\mathrm{CD} 13^{+}$, $\mathrm{CD} 14^{+}$, and $\mathrm{CD} 15^{+}$data sets), $\mathrm{B}\left(\mathrm{CD} 19^{+}\right)$and $\mathrm{T}$ cells $\left(\mathrm{CD}^{+}\right)$. Almost every cell clone marked by a specific IS accounted for only a fraction of a percent of the total clones at any given time. A few IS-marked cell clones showed a higher percentage at one time point but then disappeared or were strongly reduced at later time points (Fig. 4, $\mathrm{B}$ and $\mathrm{C}$, and fig. S16). From these results, we conclude that no clonal dominance events occurred in these patients.

To determine whether other hallmarks of insertional mutagenesis were present in the patients, we assessed the occurrence of common insertion sites (CISs), insertional hotspots that may result from integration bias at the time of transduction or in vivo selection of clones harboring integrations that confer growth advantage. CISs were identified with an algorithm based on Abel et al. (30) and by the Grubbs test for outliers (29). Both analyses showed that the MLD CISs were mostly distributed in clusters within gene-dense regions heavily targeted by LV integrations (Fig. 5A, figs. S17 to S19, and tables S15 and S16), many of which had already been described in the ALD HSC-GT trial (Fig. 5B) $(17,29)$. Given the larger size of the MLD data set, it contained almost the entire ALD CIS data set. Moreover, the Grubbs test for outliers when applied to the genomic regions surrounding each CIS confirmed that the CIS from the MLD clinical trial were not significantly over-targeted with respect to the neighboring genes, with OPTC being the only exception (fig. S19F and table S16). Cell clones with IS targeting CIS genes did not represent the most 
abundant IS-marked cell clones at any given time point of the follow-up. IS targeting OPTC were not detected in samples from the longest followup time point.

\section{Extensive HSC Gene Marking in Vivo}

To assess HSC gene marking in vivo, we analyzed the subset of IS shared among three data sets representing progenitors and mature myeloid and mature lymphoid cells in each patient. After stringent filtering to reduce the false discovery rate due to the impurity of each cell fraction analyzed and the occurrence of collisions during IS processing (fig. S20 and table S17), a fraction of ISs were consistently shared among the three data sets of each patient (Fig. 6A). This fraction in- creased with follow-up time (Fig. 6B). We then plotted the occurrence of shared ISs in each subset of cells over time, considering the $\mathrm{B}$ and $\mathrm{T}$ cells separately, and ranked them for multiple hits (Fig. 6C and table S18). The increasing presence over time of $\mathrm{CD} 34^{+}$progenitors and mature cells of myeloid and lymphoid lineages marked by identical integrations is evidence of self-renewal
Fig. 4. LV genomic integration profile. (A) Gene ontology (GO) analysis on ISs from three patients with MLD and two patients with ALD who had been treated with HSC-GT (17). This analysis was performed using the GREAT software (http/// bejerano.stanford.edu/great/). The genomic positions of ISs in each data set were weighted by the binomial test in order to evaluate the clustering at given genomic regions and by the hypergeometric test in order to evaluate the overrepresentation of gene functions. GO classes were considered significant when both tests provided a false discovery rate of $<0.05$. To compare the level of similarity between the gene classes preferentially targeted by LV integrations in ALD and MLD clinical trials, we counted the number of shared genes contained in the significantly overrepresented gene classes for GO molecular functions of each clinical trial (results for GO biological processes are shown in fig. S13). The extent of gene sharing between the overrepresented gene classes are indicated by a color scale. (B) Box plot of the percentage of sequence reads ( $y$ axis) for unequivocally mapped ISs from patients MLD01 and MLD02 in different cell types (CD34 ${ }^{+}$, myeloid, or lymphoid cells) from different sources (BM or PB) and time points (months after gene therapy, indicated below the $x$ axis). The number of reads for each IS was normalized to the total number of sequence reads from the same time point and source. ISs over the 95 percentile of the data set are shown as dots distinct from the box and whiskers, which are mostly flattened to the bottom of the plot. The total number of ISs for each lineage and time point is shown on top. Most represented integrations (with the hit gene indicated next to the dot) are enriched in oligoclonal populations, such as in PB-derived B or T cells at early time points. (C) Follow-up of the most represented ISs during time in different cell types. Percentages of sequence reads for each IS are calculated on the total number of sequence reads from the
A

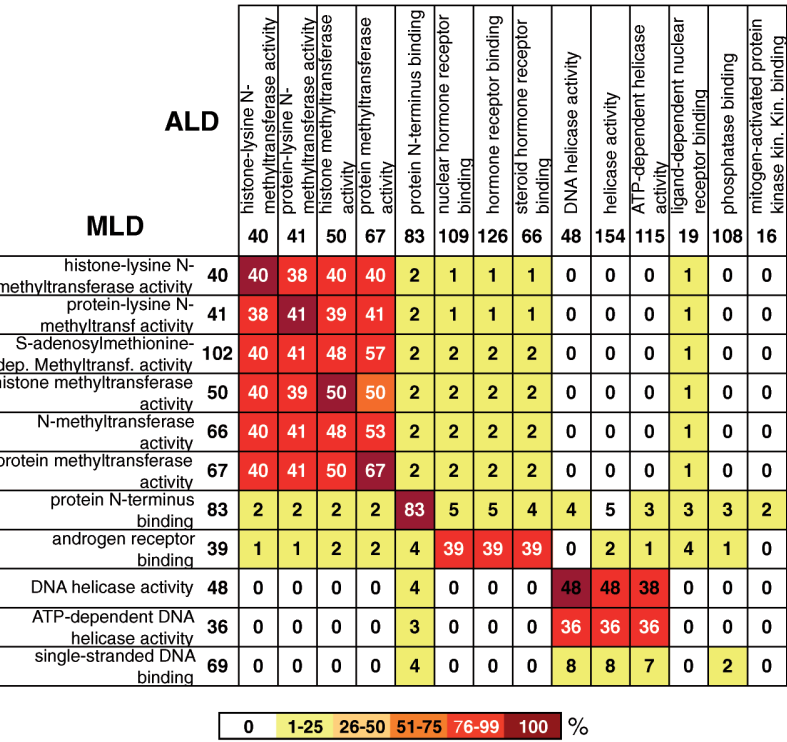

B

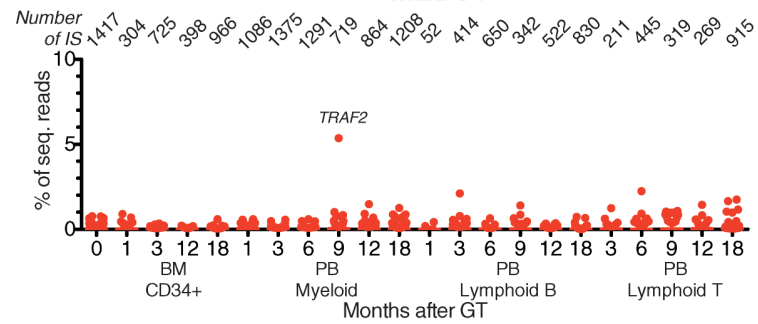

MLD02

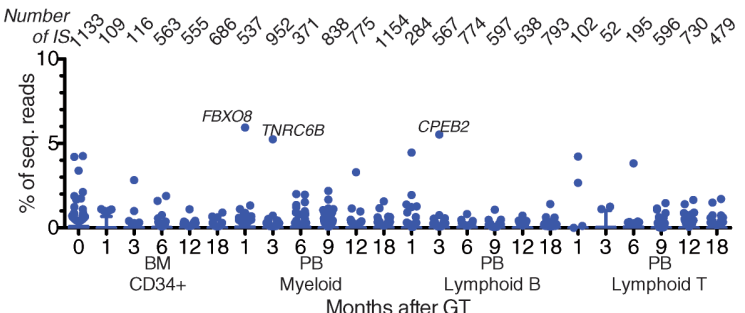

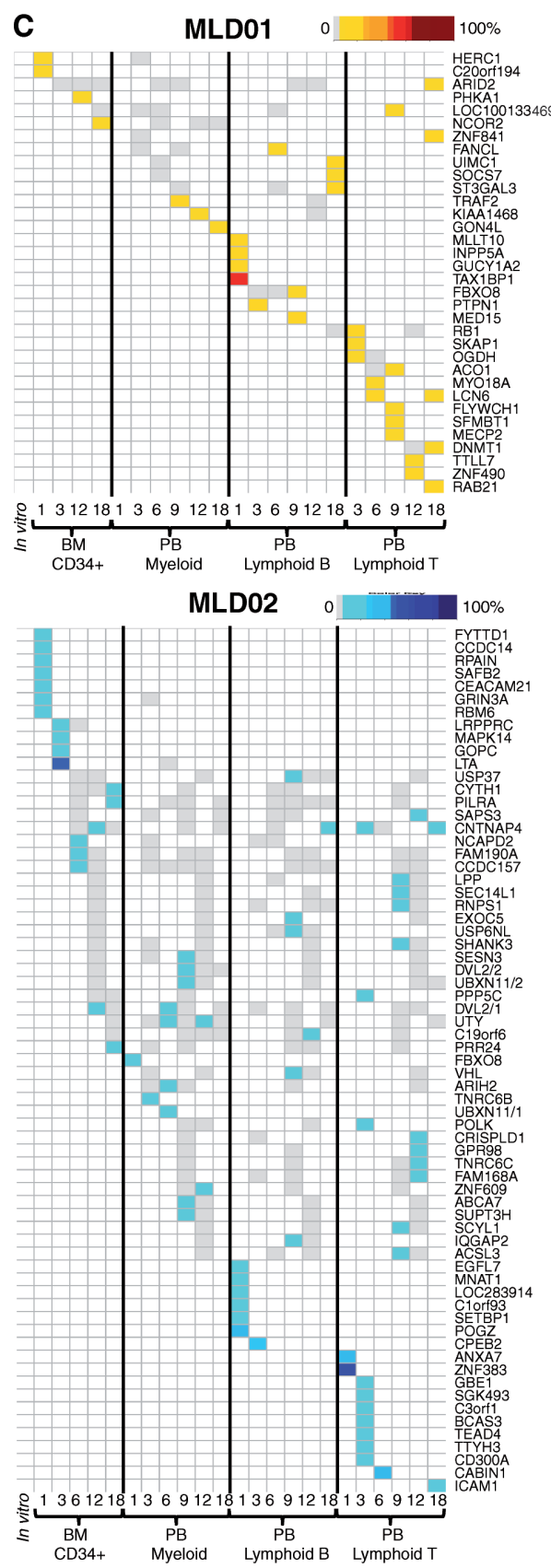

same lineage so as to emphasize the maximum relative contribution, thus obtaining values that are greater or equal to percentages shown in (B). For each IS, colored cells indicate retrieval at $\geq 5 \%$, with higher color intensity indicating higher percentage, whereas gray cells indicate retrieval at low percentage (from $0.006 \%$ to $<5 \%$ ). Lack of color indicates that the integration was not retrieved at the indicated time point and source. The targeted genes are indicated. Almost all integrations reached $\geq 5 \%$ at only one time point. 
and multilineage potential of the transduced engrafted HSCs.

To estimate the number of HSCs contributing to hematopoietic reconstitution, we used the fraction of ISs shared between independent samplings of the same cell population (supplementary materials, materials and methods). For this analysis, we considered the output of short-lived mature myeloid cells in the PB long-term after HSC-GT as readout of total HSC activity at that time. We compared ISs shared between $\mathrm{CD} 14^{+}$and $\mathrm{CD} 15^{+}$ PB cells at months 9, 12, and 18 after HSC-GT by the mark-recapture approach using the Petersen/ Schnabel estimator method (31) and Chao Poisson regression model (table S19). The resulting lower bound population size estimates were $3.7 \times 10^{3}$ to $5.7 \times 10^{3}$ in patient MLD01 and $2.1 \times 10^{3}$ to $3.4 \times 10^{3}$ in MLD02, indicating an abundant pool of engrafted transduced self-renewing progenitors in both patients.

To quantify clonal diversity in each lineage over time, we calculated the Shannon Diversity Index (Fig. 6D and fig. S22). This index measures the entropy of an IS data set taking into account the total number of ISs and their relative contribution. The diversity of $\mathrm{CD} 34^{+}$progenitors was lower early after HSC-GT as compared with that of the infused cell population and then in- creased and stabilized. PB myeloid cells showed high and stable diversity, which is consistent with that of the progenitor pool, whereas B and T cell diversity increased with time and stabilized 6 months after gene therapy. Patient MLD03 showed lower IS sharing between lineages and decreasing diversity of myeloid cells as compared with the other two patients, possibly because of the shorter follow-up and the higher $\mathrm{VCN}$ of the infused cells.

Overall, these data provide evidence that efficient ex vivo gene transfer was followed by substantial engraftment and sustained clonogenic activity of the transduced HSCs in the patients, resulting in extensive polyclonal reconstitution of hematopoiesis with gene-corrected cells.

\section{Discussion}

LI MLD is a devastating disease that invariably leads to death of affected children within a few years of symptom onset. We have shown that HSCGT halted disease manifestation and/or progression in three presymptomatic children with LI-MLD for follow-up times ranging from 18 to 24 months after treatment ( 7 to 21 months after predicted disease onset).

The clinical benefit observed in the three patients was accompanied by measurable objective findings ascertained through brain imaging and electrophysiological and biochemical studies and is in contrast with the disease evolution observed in (i) the patients' older affected siblings with matched ARSA mutations and related genetic background and (ii) our historical cohort of untreated LI-MLD patients. MRI analysis detected a minor demyelinating lesion in the brain of MLD01 1 year after treatment. This minor lesion, which might have arisen before the infused cells reached a sufficient CNS engraftment to exert beneficial effects, remained stable at all subsequent followups. This finding is clearly different from what is observed in untreated LI-MLD patients, who show rapidly progressive and widespread brain demyelination. The severe peripheral neuropathy already present in MLD01 at the time of HSC-GT improved after treatment, with amelioration of the amplitude of motor action potentials and of the nerve conduction velocities after treatment. These findings were associated with clinical evidence of normal cognitive evolution and continuous improvements in motor skills. Although the follow-up is shorter for MLD02 and MLD03, these patients remained asymptomatic at a chronological age when their disease was expected to have manifested.

In previous work with the MLD mouse model, we showed that the therapeutic benefit of

A Chr

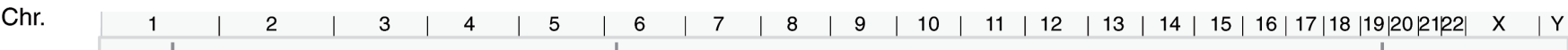
RefSeq
Genes

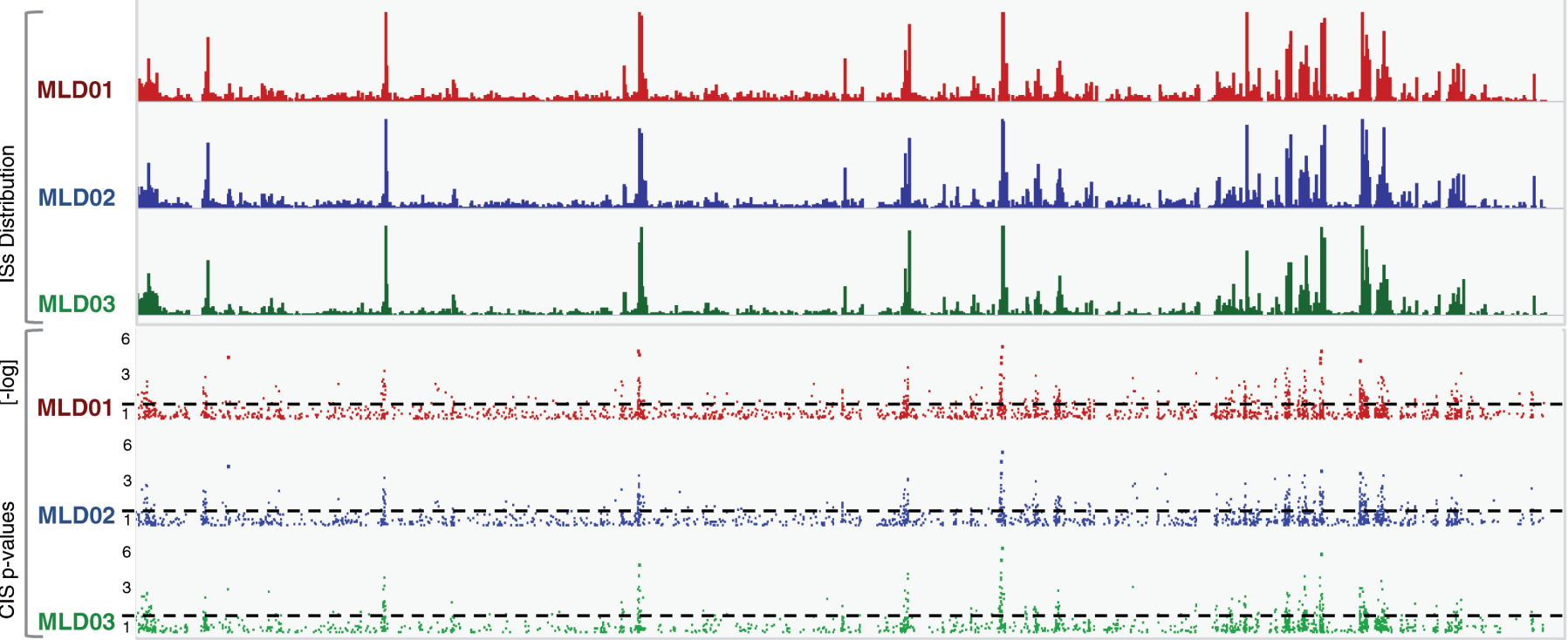

B MLD

330

ALD

Fig. 5. Common insertion site analysis. (A) Frequency distribution along chromosomes (top track) of RefSeq genes (second track below), LV integrations for each patient (third, fourth, and fifth track below for MLD01, MLD02, and MLD03, respectively). The last three tracks at the bottom represent Manhattan plots of the $P$ values of the gene integration frequencies evaluated with the genome-wide Grubbs test for outliers. The dashed lines indicate the upper limit required to reach CIS significance. CISs are enriched in gene dense regions targeted at high frequency by means of LV integrations. (B) Venn diagrams showing the overlap between CIS genes in the MLD and ALD HSC-GT trials. 
HSC-GT arises from the homing of ARSAoverexpressing HSPCs and/or their myeloid progeny to the CNS and PNS, where they contribute to the replacement of a substantial fraction of microglia/resident macrophages, scavenge the stored sulfatide, and establish a local source of functional enzyme bioavailable throughout the neural tissues. Although we could not directly probe our patients for the migration of gene-modified cells into the CNS, we documented that CSF samples from the patients contained substantial levels of ARSA protein and activity long after HSC-GT. This objective finding, together with the halted progression of neural disease, suggests that the CNS and PNS were seeded by gene-corrected myeloid cells upon HSC-GT.

Recent studies in mice have shown that microglia mostly originates from an early seeding of neural tissues by embryonic lineage macrophages followed by in situ turnover under homeostatic conditions (32). However, substantial replacement of microglia with donor-derived cells was shown after myeloablation and intravenous infusion of HSPCs $(8,33,34)$. The latter findings are consistent with clinical evidence that HSCT can provide therapeutic benefit in certain lysosomal storage diseases affecting the CNS, and with the few available studies investigating the donor origin of microglia cells within the brains of transplanted humans $(35,36)$. We speculate that high-level engraftment of the myeloid compartment in PB and BM by the transduced HSPCs is likely needed to support their efficient migration to the neural tissues. It is possible, however, that myeloid progenitors, rather than mature monocytes, are primarily responsible for seeding the CNS in our patients (34).

At least three factors may have contributed to the high levels of gene replacement observed in patients' hematopoiesis. First, we optimized the LV-manufacturing process, aiming to produce vector with high titer, infectivity, and purity using stringent lot-release criteria so as to ensure efficient transduction while limiting detrimental effects on HSPCs. Second, we validated the transduction protocol for reproducible high-rate gene transfer into BM-derived mouse-repopulating human HSPCs and infused fresh cells after transduction to avoid incurring in any cell loss associated with freeze/thawing. Last, we administered a doseadjusted myeloablative regimen before HSPC infusion to foster engraftment.

Another key goal of our study was to assess the long-term safety of HSC gene transfer. Using deep-sequencing and bioinformatics, we found no evidence that cell clones harboring ISs undergo in vivo expansion or selection. Rather, our data suggest that the genomic distribution of IS in patients' hematopoietic cells reflects the LV integration in the infused HSPCs. These findings are in sharp contrast with those reported at comparable times after HSC-GT in some other trials using $\gamma-\mathrm{RV}$; in those trials, cell clones harboring
A

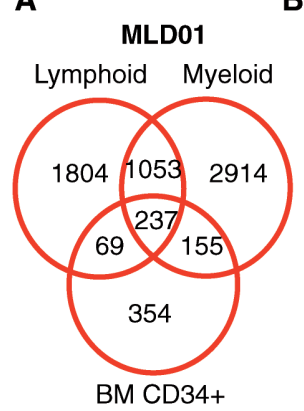

MLD02

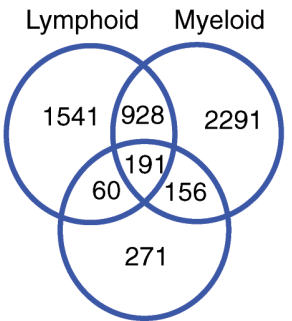

$\mathrm{BM}$ CD34+

MLD03

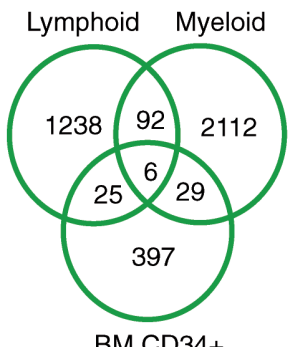

BM CD34+

B

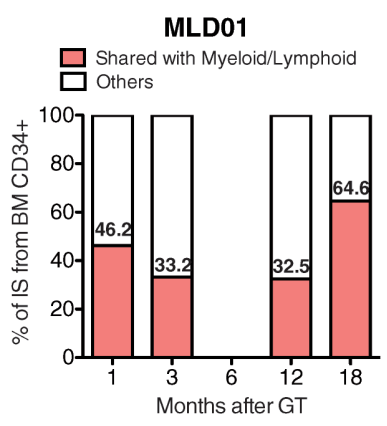

MLD02

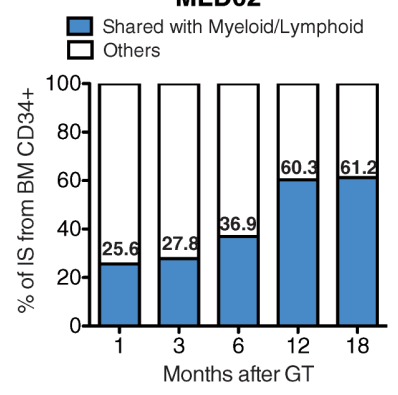

MLD03

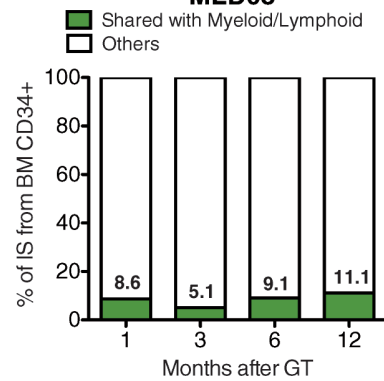

C

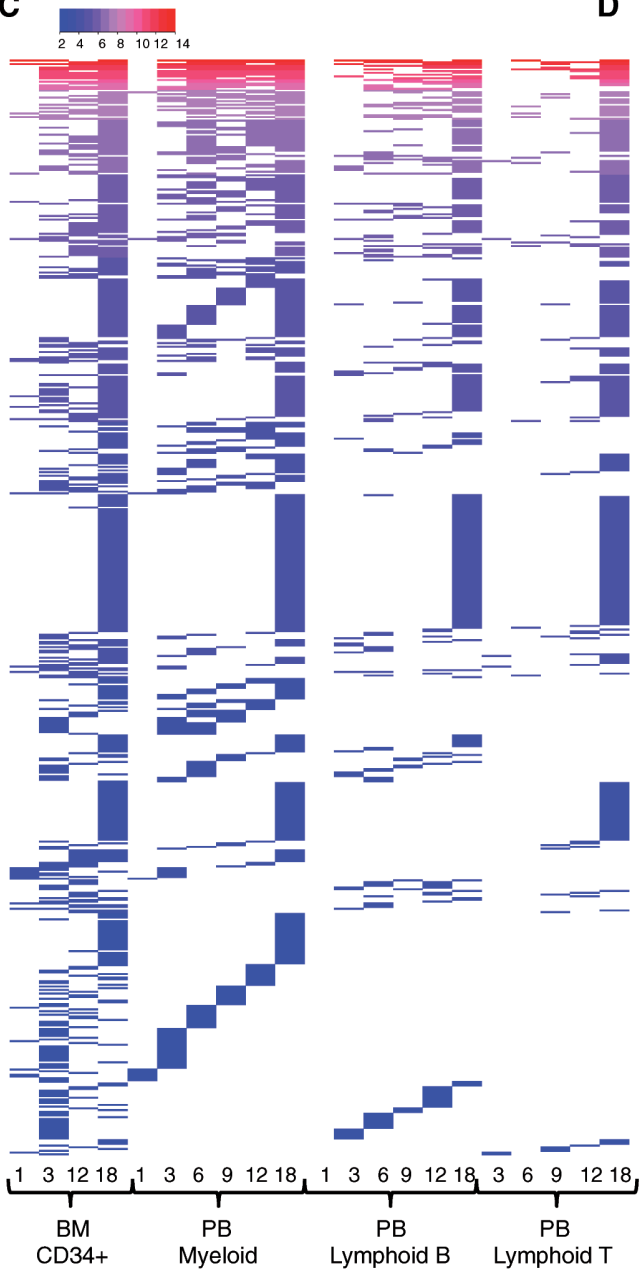

D
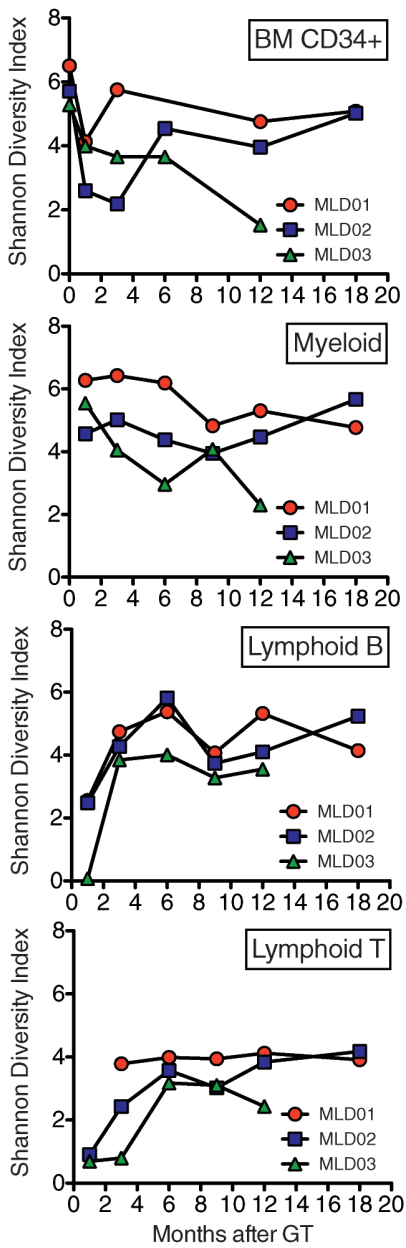

Fig. 6. Stem cell marking and clonal dynamics. Analysis of IS data was performed after filtering for collision and sample impurity. (A) Venn diagrams showing the sharing of ISs between $\mathrm{CD}^{+} 4^{+}$cells and lymphoid and myeloid lineages. (B) Percentage of shared ISs ( $y$ axis) between BM-derived $\mathrm{CD}_{4} 4^{+}$and myeloid or lymphoid lineages during time (months after GT, indicated below). (C) Tracking of ISs shared between multiple lineages with time in patient MLD01. Each

row represents a specific IS, with colored bars indicating retrieval from the indicated cell lineage and time point after gene therapy (columns). The line color varies with the degree of sharing among lineages (red, high level of sharing; blue, low level of sharing; white, no integration retrieved). PB, peripheral blood; B, B cells; T, T cells; Myelo, myeloid cells. (D) Shannon Diversity Index (y axis) was calculated to measure the clonal diversity of hematopoietic reconstitution during time. 
ISs that target oncogenes underwent expansion or selection in vivo and eventually progressed to leukemia $(14,18,37-40)$. The LV IS distribution in MLD patients overlaps with that reported for ALD patients after LV-based HSC-GT, a reassuring finding considering that no adverse effect of gene transfer has been reported in that trial after more than 5 years of follow-up. A similar integration profile in the absence of clonal expansion was observed in three patients with WiskottAldrich Syndrome treated by LV-based HSC-GT with a follow-up of up to 2.5 years (47). Overall, these data strongly support the improved safety of LV comprising self-inactivating long terminal repeat and moderately active internal promoters, which makes it unlikely that an insertion would activate a nearby oncogene $(19,20,41)$. Nevertheless, we will continue longer-term evaluation of the treated and additional patients for full assessment of the risk-benefit ratio and therapeutic potential of HSC-GT.

The stability of gene marking together with the identification of several ISs shared among progenitors, mature myeloid cells, and both major lymphoid lineages long-term after HSC-GT provide evidence that bona fide HSCs were transduced. Furthermore, the estimated large number of active HSCs would imply that a good fraction of harvested HSCs were transduced and long-term engrafted the patients. These data position LV gene transfer as a feasible means to engineer human hematopoiesis to its near entirety, an approach that may be exploited to develop novel treatments for several inherited and acquired diseases.

\section{Materials and Methods}

\section{Clinical Protocol and Patients}

The Istituto Superiore di Sanità and the Ethical Committee of the San Raffaele Scientific Institute in Milan approved the study. The study promoter is TIGET, San Raffaele Hospital, Italy and the financial sponsor of the study is the Telethon Foundation. The medicinal product received Orphan Drug Designation (ODD) (EMEA/OD/ 102/06) by the European Medicines Agency (EMA) for the treatment of MLD.

We enrolled and treated three presymptomatic LI-MLD patients, who were classified as LI according to the disease in their affected older siblings using the historical classification based on age at symptom onset (table S3). The patients were from Lebanon, the United States, and Egypt. Upon obtaining informed consent, their diagnosis was molecularly and biochemically confirmed. In all patients, the definitive MLD diagnosis was based on pathologic low ARSA activity and the presence of disease-causing ARSA gene mutations on both alleles. Presymptomatic condition at enrollment was defined on the basis of a normal neurological and cognitive examination, independently from the presence of abnormalities at neurophysiological tests or brain imaging.

Details on clinical study enrollment criteria, endpoints, and study plan can be found at
ClinicalTrials.gov \#NCT01560182. The parents of all subjects provided written informed consent for experimental treatment. Biological samples were obtained from MLD patients - healthy children and adults as controls - with approval of the San Raffaele Scientific Institute's Ethics Committee and consent from parents or subjects.

\section{Neurological and Motor Evaluation}

A standard neurological evaluation was performed in all patients for assessing the presence of disease clinical signs. In addition, we applied GMFM $(26,42)$ and the Gross Motor Function Classification for MLD (GMFC-MLD) (27) for proper quantification of patients' motor abilities. The same trained personnel applied the scales at each evaluation.

\section{Neuropsychological Evaluation}

Neuropsychological evaluation was performed by using the Bayley Scale for Infant and Toddler Development (BSID-II) (43), applied at baseline evaluation and then every 6 months.

\section{Neurophysiological Evaluation}

Sensory conduction of sural and median nerves, and motor conduction of deep peroneal and ulnar nerves were studied and a $z$ score for $\mathrm{NCV}$ was calculated for each examined nerve (patient's $\mathrm{NCV}$ - normal donors' mean NCV/normal donors' NCV standard deviation); an NCV index was calculated as the average of $z$ scores obtained from the four tested nerves for each patient per time, as described (26).

\section{MR Examination}

All children underwent anatomical MRI on a 1.5 Tesla scanner using a six-channel SENSE head coil (Gyroscan Intera, Philips, Netherlands). MRI scans were performed with Spin Echo T1 weighted images, repetition time (TR) $600 \mathrm{~ms}$, echo time (TE) $15 \mathrm{~ms}$, rec matrix 288, 22 slices, 5 and $3 \mathrm{~mm}$ thick, axial and sagittal planes; Turbo Spin Echo T2-weighted images, TE120 ms, TR $5500 \mathrm{~ms}$ 24 slices, axial, sagittal and coronal planes, 4.5 and $3 \mathrm{~mm}$ thick; fluid-attenuated inversion recovery Turbo Spin Echo, TE 140 ms, 24 slices, $4.5 \mathrm{~mm}$ thick; and volumetric T1 sequence (fast field echo, 110 slices, $1.4 \mathrm{~mm}$ thick, and $0 \mathrm{~mm}$ gap). MR examinations were performed under sedation by a senior experienced pediatric anesthesiologist (44); sedation was induced with intravenous $1 \mathrm{mg} / \mathrm{kg}$ propofol and maintained with $4 \mathrm{mg} / \mathrm{kg} /$ hour propofol continuous infusion. Throughout MRI examination, saturation of peripheral oxygen $\left(\mathrm{SpO}_{2}\right)$ was monitored with a MRI-compatible device (Magnitude, Invivo Corporation, Orlando, Florida). Desaturation $\left(\mathrm{SpO}_{2}<\right.$ $95 \%$ ) was recorded together with any adverse event requiring the intervention of the attending anesthesiologist.

\section{Large-Scale Lentiviral Vector Production}

The vector used in this study (pCCLsin.cPPT. hPGK.hARSA.WPREmut6 - PGK.ARSA.LV) is a self-inactivating lentiviral vector produced with a third-generation split packaging system and pseudotyped by the vesicular stomatitis virus $\mathrm{G}$ protein (VSV.G) (6). The vector lots were produced by MolMed (Milan, Italy) using a large-scale validated process. The vectors were produced following Good Manufacturing Practice (GMP) guidelines, except for initial studies of gene transfer protocol optimization, which used pre-GMP (GMP-like/large scale produced) vector. The protocol for vector production is detailed in fig. S3. Briefly, the ARSA LV was produced by means of transient four-plasmid transfection of $293 \mathrm{~T}$ cells. $293 \mathrm{~T}$ cells were derived from human embryonic kidney 293 cells with stable transfection of the temperature-sensitive SV40 T-antigen; a subclone of the 293T (from Stanford University) selected at the Salk Institute (La Jolla, California) for its highyield performance in production of lentiviral vectors through transient transfection was used for MCB establishment; a 293T MCB was generated at Genethon (Paris, France) in GMP conditions. 293 T cells from the master cell bank were expanded in T162 flasks and then in 10-tray cell factories and transiently transfected by means of calcium phosphate precipitation with four plasmids encoding for two core packaging constructs (pKLGag/pol and pKRev), the envelope construct (pK.G), and the transfer vector construct (pARSA). Twenty-four hours after removal of the transfection medium, the cell supernatant was harvested and stored at $4^{\circ} \mathrm{C}$. The culture medium was replaced, and after a further 24 hours, a second harvest was performed. The medium collected from the two harvests was pooled and filtered through $5 / 0.45-\mu \mathrm{m}$ filters so as to discard cell debris. The downstream purification process included a benzonase treatment overnight at $4^{\circ} \mathrm{C}$, followed by a diethylamino anion exchange (DEAE) chromatography step, concentration, and gel filtration in CellGro medium. The benzonase treatment was aimed at degrading plasmid DNA and host cell-derived DNA present in the clarified cell supernatant, thus facilitating their removal through subsequent purification steps. Anion exchange chromatography involved the absorption of negatively charged lentiviral particles to positively charged chromatographic support; viral particles were eluted by increasing ionic strength. This step was aimed at the removal of contaminants such as host cell proteins and serum-derived proteins such as bovine serum albumin (BSA). Gel filtration, also defined as size exclusion chromatography, relies on the inability of the large lentiviral particles to be retained by matrix pores. This step was aimed at the removal of salts and small-sized contaminants such as DNA fragments as well as at medium exchange. The resulting LV preparation, in CellGro medium, underwent one sterilizing $0.2-\mu \mathrm{m}$ filtration and aseptic filling. The purified vector preparation was stored at $-80^{\circ} \mathrm{C}$. The produced vector lots were characterized as described in table S2. Physical titer was measured by means of enzyme-linked immunosorbent assay (Perkin Elmer, Applied Biosystem, Foster City, 
California) for the detection of HIV-1 gag p24 capsid protein. Infectious titer was measured through transduction of a human $\mathrm{T}$ cell line with serial dilutions of vector and calculation of the copies of integrated vector per cell by quantitative PCR.

\section{HSC Isolation and Transduction \\ For in Vitro Experiments}

Healthy donor (HD) bone marrow CD $34^{+}$cells were purchased from Lonza (Basel, Switzerland) or obtained after written informed consent according to standard ethical procedure and with approval of the San Raffaele Institute Ethical Committee (protocol TIGET-01). CD34 ${ }^{+}$cells from three MLD patients not enrolled in the gene therapy trial — diagnosed on the basis of clinical symptoms, ARSA activity, and genetic analysis - were similarly obtained after written informed consent (protocol LDM1) at the time of diagnostic or therapeutic procedures. $\mathrm{CD} 34^{+}$cells were purified from mononuclear cells by means of positive selection with immunomagnetic beads according to the manufacturer's procedure (Miltenyi Biotec, Bergisch-Gladbach, Germany). Soon after purification or thawing, $\mathrm{CD} 34^{+}$cells were placed in culture on retronectin-coated non-tissue culturetreated wells (T100A Takara) in CellGro SCGM medium (2001 CellGenix) at a concentration of $1 \times 10^{6}$ cells $/ \mathrm{ml}$ in the presence of cytokines [interleukin-3 (IL-3, $60 \mathrm{ng} / \mathrm{ml}$ ), thrombopoietin (TPO, $100 \mathrm{ng} / \mathrm{ml}$ ), stem cell factor (SCF, $300 \mathrm{ng} / \mathrm{ml}$ ), and Flt3 ligand (Flt3-L, 300 ng/ml); PeproTech, Rocky Hill, New Jersey] for 24 hours of prestimulation. Cells were then transduced with ARSA LV [at a multiplicity of infection (MOI) of 100] for 14 hours. In the protocol that foresees two rounds of transduction, selected for clinical application, cells were washed for 10 hours in CellGro SCGM medium additioned with cytokines and underwent a second hit of transduction in the same conditions as the first. At the end of transduction, cells were counted and collected for clonogenic assays, flow cytometry, and in vivo studies. Remaining cells were plated in Iscove's modified Dulbecco's medium (IMDM) $-10 \%$ fetal bovine serum (FBS) with cytokines (IL-3, $60 \mathrm{ng} / \mathrm{ml}$; IL-6, $60 \mathrm{ng} / \mathrm{ml}$; $\mathrm{SCF}, 300 \mathrm{ng} / \mathrm{ml}$ ) and cultured for a total of 14 days. Thereafter, cells were collected for molecular and biochemical studies.

\section{For Patients' Treatment}

Please refer to fig. S2. Total bone marrow was collected from the iliac crests under sterile conditions and using general anesthesia, on day -4 , according to an internal standard operating procedure. The total collected volume was estimated according to the content of CD $34^{+}$cells evaluated by previous bone marrow aspiration; on average, 20 to $25 \mathrm{ml} / \mathrm{kg}$ patient's body weight was collected. The harvested bone marrow-collected in a dedicated bag, sealed, and identified — was then transferred to MolMed S.p.A and kept in sterile conditions in a refrigerator at $4^{\circ} \mathrm{C}$ for 24 hours. $\mathrm{CD} 34^{+}$cells were then purified on day -3 from mononuclear cells by means of positive selection with immunomagnetic beads according to the manufacturer's procedure (Miltenyi Biotec, Bergisch-Gladbach, Germany) in GMP conditions. The purified cells were cultured in retronectin-coated VueLife bags (American Fluoroseal, Gaithersburg, Maryland) in serum-free medium supplemented with GMP-grade cytokines and exposed twice to GMP-grade-purified vector at multiplicity of infection of 100, for a total culture time of 60 hours. At the end of the transduction procedure, the transduced $\mathrm{CD} 34^{+}$cells were harvested, washed with Cell Grow medium, and resuspended in saline solution at the concentration of $2 \times 10^{6}$ to $10 \times$ $10^{6} / \mathrm{ml}$ for patient's infusion. At the end of transduction, a fraction of the cells was collected for clonogenic assays, flow cytometry, and in vitro culture, as above. Cells were kept at $4^{\circ} \mathrm{C}$ up to the time of infusion, upon batch release according to quality control tests [results are available at infusion for viability, immunophenotype, endotoxin, large T Ag DNA, and mycoplasma (table S4)].

\section{Patients' Conditioning}

Patients received a total of 14 body weight-based doses of intravenous busulfan, given every 6 hours from day -4 to day -1 . The busulfan plasmatic levels were monitored with serial sampling following first and fifth infusion. The doses after the 5 th and 10th were adjusted, if needed, according to plasmatic Busulfan levels with an ideal AUC of range $4200-5600 \mu \mathrm{g} / \mathrm{L} *$ hour, target $4800 \mu \mathrm{g} / \mathrm{L} *$ hour. The interval between the end of the last intravenous busulfan dose and final product infusion was $\geq 24$ hours in order to allow safe infusion of the stem cells. At day 0 , after premedication with clorfeniramine $(0.25 \mathrm{mg} / \mathrm{kg}$, max dose $10 \mathrm{mg}$ ) or equivalent drug, the resuspended CD34 cell product was infused intravenously through the central catheter.

During hospitalization, patients were cared for in an isolation unit and received supportive therapy according to local standards.

\section{Isolation of Hematopoietic Subpopulations from Patients' Peripheral Blood and Bone Marrow}

Starting from mononuclear cells (MNCs) obtained from Lymphoprep gradient, human bone marrow and peripheral blood subpopulations were obtained through positive selection of the following markers (Miltenyi Biotec microbeads): CD34, CD13, CD15, CD56, CD19, CD3, glycophorin A (GLYA), and CD61 from the $\mathrm{BM}$, and CD15, CD14, CD56, CD19, and CD3 from $\mathrm{PB}$, according to manufacturer's procedures. Purity of each subpopulation was checked by means of flow-cytometry. Data are reported in table S11.

\section{Clonogenic Assay (CFC)}

The CFC assay was performed by means of plating in methylcellulose medium (human MethoCult; StemCell Technologies) either 800 to $1000 \mathrm{CD}^{+}$ cells $/ \mathrm{ml}$ or $1.25 \times 10^{4}$ to $1 \times 10^{6} \mathrm{MNCs} / \mathrm{ml}$ purified from BM or PB. After 14 days of culture, colonies were counted, distinguishing myeloid and erythroid origin, and plucked for molecular analysis.

\section{Quantitative PCR}

Genomic DNA was extracted from CD34 $4^{+}$liquid culture, peripheral blood mononuclear cells (PBMCs), MNCs, PB, and BM subpopulations of MLD patient samples with QIAamp DNA Blood Micro and Mini Kits (Qiagen, Hilden, Germany). Single colonies plucked from the CFC assay were digested for 4 hours at $37^{\circ} \mathrm{C}$ in Lysis Buffer (Lauryl Ether 0.1\%, TRIS/hydrochloride (HCL) $10 \mathrm{mM}, 100 \mu \mathrm{g} / \mathrm{ml}$ Proteinase K). After proteinase $\mathrm{K}$ inactivation $\left(10 \mathrm{~min}\right.$ at $\left.95^{\circ} \mathrm{C}\right)$, lysates were centrifuged at 13,000 revolutions per $\mathrm{min}$ at $4^{\circ} \mathrm{C}$ for $15 \mathrm{~min}$.

LV sequences were detected by means of quantitative PCR on 25 to $50 \mathrm{ng}$ of total genomic DNA or $10 \mu \mathrm{l}$ of lysate supernatants by using previously described primers and probe (22).

Absolute quantifications were plotted on standard curves prepared with serial dilutions of genomic DNA or cell lysate from a T cell clone containing 1 copy HIV/cell.

Engraftment of the transduced cells was evaluated by means of quantitative PCR on individual colonies from CFC assay performed on either PB- or BM-derived cells and expressed as percentage $(\%)$ of $\mathrm{LV}^{+}(\mathrm{VCN} \geq 0.5)$ colonies on total tested colonies ( $\geq 250$ cells/colony).

\section{Analysis of Vbeta Repertoire}

The analysis of Vbeta repertoire of the $\mathrm{T}$ cell receptor was performed on $50 \mu \mathrm{l}$ of patients' total $\mathrm{PB}$ according to the manufacturing procedure described in the V Beta kit; Beckman Coulter (823497) (Fullerton, California). 10000 events were acquired with FACS Canto II (BD-Biosciences, Europe) and then analyzed with FlowJo (TreeStar, Ashland, Oregon). The percentage of each Vbeta population was compared with the range of expression obtained from the analysis of a pool of 85 healthy donors provided by the manufacturer.

\section{Immunophenotype Analysis}

Analysis of the immunophenotype of patients' total PB and BM was performed by Laboraf (Ospedale San Raffaele) as described by internal SOPs.

\section{ARSA Activity Determination}

p-Nitrocatechol Sulfate (PNCS)

CD34 ${ }^{+}$liquid culture, PBMCs, MNCs, PB, and BM subpopulations of MLD patients and healthy donors were resuspended in sodium acetate trihydrate $0.05 \mathrm{M}(500,000$ cells in $25 \mu \mathrm{l})$. Samples were then sonicated and centrifuged, and the supernatant was collected for ARSA activity and protein quantification (Bradford assay; Biorad \#500-0006) (Hercules, California). ARSA activity was detected with PNCS as substrate, as described, loading a maximum concentration of 
protein extract of $0.3 \mu \mathrm{g} / \mu \mathrm{l}$ diluted in sodium acetate trihydrate $0.05 \mathrm{M}(9)$.

\section{DEAE-Cellulose Chromatography and 4-Methyl-Umbellipheryl-Sulfate}

Cells were harvested, washed in phosphatebuffered saline, and resuspended in $10 \mathrm{mM}$ TRIS/ $\mathrm{HCl}$ buffer, $\mathrm{pH} 7.5$, containing $0.1 \%$ (v/v) Nonidet NP40 detergent (Sigma-Aldrich, St. Louis, Missouri). Cell lysates were subjected to three rounds of sonication. Procedures were carried out at $4^{\circ} \mathrm{C}$. Proteins were measured with the Bradford method by using bovine serum albumin as standard.

Similar cell number $(500,000$ cells $)$ was loaded into a $0.2-\mathrm{ml}$ DEAE chromatography column equilibrated with a $25 \mathrm{mM}$ TRIS/HCl buffer, $\mathrm{pH}$ 7.5. The flow rate was $0.2 \mathrm{ml} / \mathrm{min}$. After washing with $5 \mathrm{ml}$ of $25 \mathrm{mM}$ TRIS/HCl buffer plus $50 \mathrm{mM}$ $\mathrm{NaCl}, \mathrm{pH} 7.5$, enzyme activity retained by the column was eluted by $2.5 \mathrm{ml}$ TRIS/HCl buffer plus $250 \mathrm{mM} \mathrm{NaCl}, \mathrm{pH} 7.5$ (starting from fraction n.5). Fractions $(0.5 \mathrm{ml})$ were collected and assayed for the ARSA activity by using the artificial fluorogenic substrate 4-methyl-umbellipherylsulfate (MUS) dissolved in $0.05 \mathrm{M} \mathrm{Na}$-acetate/ acetic acid buffer, $\mathrm{pH}$ 5.5. A volume of $50 \mu \mathrm{l}$ of test sample was incubated with $100 \mu \mathrm{L}$ of a $0.01 \mathrm{M}$ solution of substrate respectively in the presence and absence of $125 \mu \mathrm{M}$ AgNO3 (a specific ARSA inhibitor) at $37^{\circ} \mathrm{C}$. Enzymatic reactions were stopped by adding $0.2 \mathrm{M}$ Glycine/ $\mathrm{NaOH}, \mathrm{pH} 10.6$. Fluorescence of the liberated 4-methylumbelliferone was measured on a Perkin Elmer LS3 spectrofluorimeter (excitation $360 \mathrm{~nm}$, emission $446 \mathrm{~nm}$ ). ARSA activity was calculated by subtracting the value obtained in the presence of $\mathrm{AgNO} 3$ [arylsulfatase B (ARSB) activity] from that measured in the absence of the inhibitor (ARSA+ARSB activity) (45).

\section{Sulphatide Assay}

Sulfatide (5 mM) (Matreya, St. Pleasant Gap, Pennsylvania) was resuspended in cloroform: methanol:water (2:1:0.1), dried overnight at room temperature, and resuspended in the ARSA enzymatic buffer [100 mM Na/acetate, $\mathrm{pH} 4.5$, containing $20 \mathrm{mM} \mathrm{MnCl} 2$ and taurodeoxyxholate $(100 \mathrm{mg} / 50 \mu \mathrm{l})]$. For the reaction $(100 \mu \mathrm{l}$ total volume), $0.003 \mathrm{mU}$ ARSA were added to $21 \mathrm{nmol}$ sulfatide at $37^{\circ} \mathrm{C}$ for 24 hours. The assay was stopped with $0.85 \mathrm{ml}$ cloroform:methanol:water (1:1) plus $0.8550 \mathrm{mN}$ sulfuric acid and $0.170 \mathrm{ml}$ Azure A $[0.4 \mathrm{mg} / \mathrm{ml}]$. After a centrifugation at $300 \mathrm{~g} \times 5 \mathrm{~min}$, the lower phase was recovered, and the absorbance measured at $650 \mathrm{~nm}$ in a microplate reader (GDV DV-990BV6). The naomoles of sulfatide hydrolyzed by ARSA were calculated by subtracting the value measured in the presence of the enzyme respect to a reference assay performed in the absence of ARSA.

\section{In Situ Hybridization and Immunofluorescence}

Cells were grown on polyornithine-coated glass coverslips $(0.01 \%$ solution, Sigma-Aldrich Corp, Italy) 72 hours in IMDM 10\% FBS medium and then fixed with $4 \%$ paraformaldehyde (PFA) solution.

An antisense 598-base pair digoxigeninlabeled oligonucleotide complementary to W-Pre mRNA sequence and a control sense sequence were produced. The in situ protocol was modified from (46). Cells were treated with prehybridization protocol and incubated overnight at $37^{\circ} \mathrm{C}$ with $0.5 \mu \mathrm{g} / \mathrm{ml}$ probe. The probe was detected by sheep anti Digoxigenin 1:500 (Roche Applied Science, Penzberg, Germany) overnight $4^{\circ} \mathrm{C}$.

Immunofluorescence on ARSA-hemagglutinin (HA) (9)-transduced Hela cells and on patients' and healthy donors' PB cells or HeLa cells was performed overnight at $4^{\circ} \mathrm{C}$ by using rabbit antiHuman Lamp1 1:100 (Abcam), goat anti-Human ARSA 1:50 (Abnova), and/or rat anti-HA 1:100 (Roche Applied Science). Secondary antibodies were incubated 1:500 for 1 hour at RT as follows: donkey anti-sheep Alexa488, donkey anti-goat Alexa546, donkey anti-rat Alexa488, and donkey anti-rabbit Alexa647. Nuclei were counterstained with 4',6-diamidino-2-phenylindole. Images were acquired with Leica (Solms, Germany) TCS SP2 laser scanning confocal with $63 \times$ oil objective.

\section{$\operatorname{Rag2}^{-1-} I 12 r \gamma^{-1-}$ Mice Transplantation and Engraftment Evaluation}

Rag2 ${ }^{-/-}$Il2 $\mathrm{ry}^{-/-}$mice were obtained from the Central Institute for Experimental Animals, Nogawa, Japan and maintained in our animal facility according to approved protocols. Three-day-old mice were sublethally irradiated $\left(40,000 \mu \mathrm{Gy} / \mathrm{m}^{2}\right) 24$ hours before intravenous injection of untransduced and unmanipulated or transduced $\mathrm{CD} 34^{+}$cells. Eight to 12 weeks after the transplantation, mice were killed and hematopoietic organs (bone marrow, spleen, and thymus) were collected after intracardiac PBS perfusion and processed for fluorescence-activated cell sorting (FACS) analysis. All procedures were performed according to protocols approved by the Animal Care and Use Committee of the San Raffaele Scientific Institute (IACUC \#325) and communicated to the Ministry of Health and local authorities according to Italian law.

\section{Flow Cytometry}

Patients'samples - subpopulation analysis

From each sample, 5000 cells were washed and resuspended in blocking buffer (PBS with 5\% FBS 2\% BSA Fc Block 1:100), then $1 \mu 1$ of the following human-specific conjugated antibodies were added: APC-conjugated anti-CD56, PE-conjugated anti-CD34, anti-CD13 and antiglycophorinA, TC-conjugated anti-CD19, and antiCD3, FITC-conjugated anti-CD15 and anti-CD61, PB-conjugated anti-CD14 under unstained control (DAKO, Denmark; BD-Pharmigen, Europe).

\section{Rag2 ${ }^{-/}$IL $2 \mathrm{rr}^{-/}$mice - engraftment evaluation}

From each sample, 250,000 cells were washed and resuspended in blocking buffer (PBS with $5 \%$ FBS $2 \%$ BSA Fc Block 1:100), then $0.5 \mu$ l of the following human-specific conjugated anti- bodies were added: APC-conjugated anti-CD45, PE-conjugated anti-CD34, anti-CD13, anti-CD4, anti-CD11b and TC-conjugated anti-CD19, anti$\mathrm{CD} 8$, anti-CD3 under control of relative IgG isotype control (DAKO; BD-Biosciences).

After $20 \mathrm{~min}$ on ice, cells were washed, resuspended in PBS 2\% FBS, 1\% PFA for cytometric analysis. Of 100,000 events, 3000 were acquired with FACS Canto II (BD-Biosciences, Europe) and then analyzed by FlowJo (TreeStar, Ashland, Oregon). During quadrant analysis, only fluorescence that excluded $>99.9 \%$ of isotype control events was considered to be specific.

\section{Vector Integration Site Analysis}

A detailed description of the materials and methods used for vector integration site analysis is reported in the supplementary materials, materials and methods section.

\section{References and Notes}

1. ]. Aicardi, Diseases of the Nervous System in Childhood (Mac Keith Press, London, ed. 2, 1998).

2. A. Biffi, G. Lucchini, A. Rovelli, M. Sessa, Metachromatic leukodystrophy: An overview of current and prospective treatments. Bone Marrow Transplant. 42, S2-S6 (2008). doi: 10.1038/bmt.2008.275; pmid: 18978739

3. A. Biffi, P. Aubourg, N. Cartier, Gene therapy for leukodystrophies. Hum. Mol. Genet. 20, R42-R53 (2011). doi: 10.1093/hmg/ddr142; pmid: 21459776

4. W. Krivit, ]. H. Sung, E. G. Shapiro, L. A. Lockman, Microglia: The effector cell for reconstitution of the central nervous system following bone marrow transplantation for lysosomal and peroxisomal storage diseases. Cell Transplant. 4, 385-392 (1995). doi: 10.1016/0963-6897(95)00021-0; pmid: 7582569

5. A. M. Rovelli, C. G. Steward, Hematopoietic cell transplantation activity in Europe for inherited metabolic diseases: Open issues and future directions. Bone Marrow Transplant. 35, S23 (2005). doi: 10.1038/sj.bmt.1704839

6. J. J. Boelens, V. K. Prasad, J. Tolar, R. F. Wynn, C. Peters, Current international perspectives on hematopoietic stem cell transplantation for inherited metabolic disorders. Pediatr. Clin. North Am. 57, 123-145 (2010) doi: 10.1016/j.pcl.2009.11.004; pmid: 20307715

7. J. J. Boelens, Trends in haematopoietic cell transplantation for inborn errors of metabolism. J. Inherit. Metab. Dis. 29, 413-420 (2006). doi: 10.1007/s10545-005-0258-8; pmid: 16763911

8. A. Biffi et al., Correction of metachromatic leukodystrophy in the mouse model by transplantation of genetically modified hematopoietic stem cells. J. Clin. Invest. 113, 1118-1129 (2004). pmid: 15085191

9. A. Biffi et al., Gene therapy of metachromatic leukodystrophy reverses neurological damage and deficits in mice. J. Clin. Invest. 116, 3070-3082 (2006) doi: 10.1172/]CI28873; pmid: 17080200

10. I. Visigalli et al., Gene therapy augments the efficacy of hematopoietic cell transplantation and fully corrects mucopolysaccharidosis type I phenotype in the mouse model. Blood 116, 5130-5139 (2010). doi: 10.1182/ blood-2010-04-278234; pmid: 20847202

11. B. Gentner et al., Identification of hematopoietic stem cell-specific miRNAs enables gene therapy of globoid cell leukodystrophy. Sci. Transl. Med. 2, 58ra84 (2010). doi: 10.1126/scitranslmed.3001522; pmid: 21084719

12. A. Aiuti et al., Gene therapy for immunodeficiency due to adenosine deaminase deficiency. N. Engl. J. Med. 360, 447-458 (2009). doi: 10.1056/NEJMoa0805817; pmid: 19179314

13. M. Cavazzana-Calvo, A. Fischer, Gene therapy for severe combined immunodeficiency: Are we there yet? J. Clin. Invest. 117, 1456-1465 (2007). doi: 10.1172/]CI30953; pmid: 17549248 
14. M. G. Ott et al., Correction of X-linked chronic granulomatous disease by gene therapy, augmented by insertional activation of MDS1-EVI1, PRDM16 or SETBP1. Nat. Med. 12, 401-409 (2006). doi: 10.1038/nm1393; pmid: 16582916

15. L. Naldini, Ex vivo gene transfer and correction for cell-based therapies. Nat. Rev. Genet. 12, 301-315 (2011). doi: 10.1038/nrg2985; pmid: 21445084

16. L. Naldini et al., In vivo gene delivery and stable transduction of nondividing cells by a lentiviral vector. Science 272, 263-267 (1996). doi: 10.1126/ science.272.5259.263; pmid: 8602510

17. N. Cartier et al., Hematopoietic stem cell gene therapy with a lentiviral vector in X-linked adrenoleukodystrophy. Science 326, 818-823 (2009). doi: 10.1126/ science.1171242; pmid: 19892975

18. S. Hacein-Bey-Abina et al., Insertional oncogenesis in 4 patients after retrovirus-mediated gene therapy of SCID-X1. J. Clin. Invest. 118, 3132-3142 (2008). doi: $10.1172 / \mathrm{Cl} 35700$; pmid: 18688285

19. E. Montini et al., Hematopoietic stem cell gene transfer in a tumor-prone mouse model uncovers low genotoxicity of lentiviral vector integration. Nat. Biotechnol. 24 687-696 (2006). doi: 10.1038/nbt1216; pmid: 16732270

20. E. Montini et al., The genotoxic potential of retroviral vectors is strongly modulated by vector design and integration site selection in a mouse model of HSC gene therapy. J. Clin. Invest. 119, 964-975 (2009). doi: 10.1172/]Cl37630; pmid: 19307726

21. A. Capotondo et al., Safety of arylsulfatase A overexpression for gene therapy of metachromatic leukodystrophy. Hum. Gene Ther. 18, 821-836 (2007). doi: 10.1089/ hum.2007.048; pmid: 17845130

22. S. Scaramuzza et al., Preclinical safety and efficacy of human $\mathrm{CD}_{3} 4^{+}$cells transduced with lentiviral vector for the treatment of Wiskott-Aldrich syndrome. Mol. Ther. 21, 175-184 (2013). doi: 10.1038/mt.2012.23; pmid: 22371846

23. R. L. Koul et al., Late infantile metachromatic leucodystrophy in two siblings. Indian Pediatr. 31, 694-698 (1994).pmid: 7896397

24. S. Yatziv, A. Russell, An unusual form of metachromatic leukodystrophy in three siblings. Clin. Genet. 19, 222-227 (1981). doi: 10.1111/j.1399-0004.1981.tb00702.x pmid: 6115727

25. T. Satoh et al., Metachromatic leukodystrophy. Report of siblings with the juvenile type of metachromatic leukodystrophy. Acta Pathol. Jpn. 38, 1041-1051 (1988). pmid: 3188912

26. A. Biffi et al., Metachromatic leukodystrophy-mutation analysis provides further evidence of genotype-phenotype correlation. Clin. Genet. 74, 349-357 (2008). doi: 10.1111/j.1399-0004.2008.01058.x; pmid: 18786133

27. C. Kehrer, G. Blumenstock, C. Raabe, I. Krägeloh-Mann, Development and reliability of a classification system for gross motor function in children with metachromatic leucodystrophy. Dev. Med. Child Neurol. 53, 156-160 (2011). doi: 10.1111/j.1469-8749.2010.03821.x pmid: 21087233

28. S. Groeschel et al., Metachromatic leukodystrophy: Natural course of cerebral MRI changes in relation to clinical course. J. Inherit. Metab. Dis. 34, 1095-1102 (2011). doi: 10.1007/s10545-011-9361-1; pmid: 21698385

29. A. Biffi et al., Lentiviral vector common integration sites in preclinical models and a clinical trial reflect a benign integration bias and not oncogenic selection. Blood 117 5332-5339 (2011). doi: 10.1182/blood-2010-09-306761; pmid: 21403130
30. U. Abel et al., Real-time definition of non-randomness in the distribution of genomic events. PLOS ONE 2, e570 (2007). doi: 10.1371/journal.pone.0000570; pmid: 17593969

31. G. P. Wang et al., Dynamics of gene-modified progenitor cells analyzed by tracking retroviral integration sites in a human SCID-X1 gene therapy trial. Blood $\mathbf{1 1 5}$ 4356-4366 (2010). doi: 10.1182/blood-2009-12-257352; pmid: 20228274

32. F. Ginhoux et al., Fate mapping analysis reveals that adult microglia derive from primitive macrophages. Science 330, 841-845 (2010). doi: 10.1126/ science.1194637; pmid: 20966214

33. ]. Priller et al., Targeting gene-modified hematopoietic cells to the central nervous system: Use of green fluorescent protein uncovers microglial engraftment. Nat. Med. 7, 1356-1361 (2001). doi: 10.1038/nm1201-1356; pmid: 11726978

34. A. Capotondo et al., Brain conditioning is instrumental for successful microglia reconstitution following hematopoietic stem cell transplantation. Proc. Natl. Acad. Sci. U.S.A. 109, 15018-15023 (2012). doi: 10.1073/pnas.1205858109; pmid: 22923692

35. K. Araya et al., Localized donor cells in brain of a Hunter disease patient after cord blood stem cell transplantation. Mol. Genet. Metab. 98, 255-263 (2009). doi: 10.1016/j. ymgme.2009.05.006; pmid: 19556155

36. K. Allers et al., Evidence for the cure of HIV infection by CCR5 $32 / \Delta 32$ stem cell transplantation. Blood 117 2791-2799 (2011). doi: 10.1182/blood-2010-09-309591; pmid: 21148083

37. S. J. Howe et al., Insertional mutagenesis combined with acquired somatic mutations causes leukemogenesis following gene therapy of SCID-X1 patients. J. Clin. Invest. 118, 3143-3150 (2008). doi: 10.1172/JCI35798; pmid: 18688286

38. S. Hacein-Bey-Abina et al., A serious adverse event after successful gene therapy for $\mathrm{X}$-linked severe combined immunodeficiency. N. Engl. J. Med. 348, 255-256 (2003). doi: 10.1056/NEJM200301163480314; pmid: 12529469

39. S. Hacein-Bey-Abina et al., LMO2-associated clonal T cell proliferation in two patients after gene therapy for SCID-X1. Science 302, 415-419 (2003). doi: 10.1126/ science.1088547; pmid: 14564000

40. S. Stein et al., Genomic instability and myelodysplasia with monosomy 7 consequent to EVI1 activation after gene therapy for chronic granulomatous disease. Nat. Med. 16, 198-204 (2010). doi: 10.1038/nm.2088; pmid: 20098431

41. D. Zychlinski et al., Physiological promoters reduce the genotoxic risk of integrating gene vectors. Mol. Ther. 16, 718-725 (2008). doi: 10.1038/mt.2008.5; pmid: 18334985

42. D. J. Russell et al., The gross motor function measure: A means to evaluate the effects of physical therapy. Dev. Med. Child Neurol. 31, 341-352 (1989). doi: 10.1111/ j.1469-8749.1989.tb04003.x; pmid: 2753238

43. R. G. Voigt et al., Concurrent and predictive validity of the cognitive adaptive test/clinical linguistic and auditory milestone scale (CAT/CLAMS) and the Mental Developmental Index of the Bayley Scales of Infant Development. Clin. Pediatr. (Phila.) 42, 427-432 (2003). doi: 10.1177/000992280304200507 pmid: 12862346

44. C. Mattioli et al., Sedation for children with metachromatic leukodystrophy undergoing MRI. Paediatr. Anaesth. 17, 64-69 (2007). doi: 10.1111/j.1460-9592.2006.02002.x; pmid: 17184435

45. S. Martino et al., Expression and purification of a human, soluble Arylsulfatase A for Metachromatic Leukodystrophy enzyme replacement therapy. ]. Biotechnol. 117 243-251 (2005). doi: 10.1016/j.jbiotec.2005.01.018; pmid: 15862354

46. G. V. Childs, In situ hybridization with nonradioactive probes. Methods Mol. Biol. 123, 131-141 (2000). pmid: 10547766

47. A. Aiuti et al., Science 341, 1233151 (2013) doi: 341,1233151

Acknowledgments: This work was supported by Fondazione Telethon (TIGET grant B2 and F3), the European Union (EU) Seventh Framework Program (FP7)-HEALTH F2-2010-241622 [Therapeutic Challenge in Leukodystrophies (LEUKOTREAT)], the European Leukodystrophy Foundation (ELA 2007-00515), and Italian Ministry of Health (Progetto Giovani Ricercatori GR-2008-57) to A.B.; Fondazione Telethon (TIGET grant D2), the EU FP7-HEALTH (GA 222878 PERSIST), the European Research Council (ERC) (Advanced Grant 249845 TARGETINGGENETHERAPY) and the Italian Ministry of Health to L.N.; Fondazione Telethon (TIGET grant D1 and core grant), Association for International Cancer Research (AICR 09-0784), and Italian Ministry of Health (Progetto Giovani Ricercatori GR-2007-684057) to E.M.; and the Italian Higher Institute of Health (Italia-USA Collaborative Program on Rare Diseases) to M.S. We thank the medical and nursing staff of the TIGET Pediatric Clinical Research Unit, Pediatric Immunohematology and Bone Marrow Transplant Unit; R. Miniero, R. Fiori, P. Silvani, and L. Sacchi for critical help with patient management; L. Callegaro, S. Di Nunzio, M. Bonopane, G. Tomaselli, and the TIGET clinical trial office for trial management; P. Massariello and other MolMed staff for help with patient cell manipulation; I. Spiga for ARSA gene mutation analysis; S. Gerevini for initial brain MRI studies; P. Aubourg for patient referral and, together with N. Cartier, for sharing the original ALD HSC-GT IS data set; D. Redaelli and S. Acquati for molecular and biochemical studies; F. Morena for biochemical studies; A. Capotondo for studies on the MLD mouse model; M. Marini and D. Cesana for technical help on LAM-PCR amplification and bioinformatics; M. Gabaldo for support with project management; G. Royal, L. King, and F. Govani for help with 454-pyrosequencing and sequence data management; and J. Appleby for advice and support. We are especially indebted to F. Pasinelli for unremitting support of this project and to the patients who joined the trial and their families for their commitment and endurance. We dedicate this work to the memory of all MLD patients and their families who accompanied us in the long path from preclinical studies to clinical testing. C.B. is Chief Executive Officer and Chairman of the Scientific Advisory Board of MolMed S.p.A., a biotechnology company focused on research, development, and clinical validation of new cancer therapies. L.N. is an inventor of several patents on lentiviral vector technology that are owned by the Salk Institute and Cell Genesis and are licensed to Lentigen. L.N. is entitled to receive royalties from one of these patents (U.S. Patent No. 6,013,516 with the Salk Institute).

\section{Supplementary Materials}

www.sciencemag.org/cgi/content/full/science.1233158/DC1

Materials and Methods

Figs. $\mathrm{S} 1$ to $\mathrm{S} 22$

Tables S1 to S19

References (48-60)

26 November 2012; accepted 25 June 2013

Published online 11 July 2013;

$10.1126 /$ science. 1233158 